\title{
Assessment of the mesh refinement influence on the computed flow-fields about a model train in comparison with wind tunnel measurements
}

\author{
K.A. Weinman ${ }^{1, *}$, M.Fragner ${ }^{2}$, R.Deiterding ${ }^{3}$, D.Heine ${ }^{1}$, U.Fey ${ }^{1}$, F.Braenstroem ${ }^{4}$, B.Schultz ${ }^{4}$, C.Wagner ${ }^{1,5}$ \\ ${ }^{1}$ German Aerospace Center (DLR), Institute of Aerodynamics and Flow Technology, Göttingen, Germany \\ ${ }^{2}$ Hochschule Hannover, Fakultät I - Elektro- und Informationstechnik, Hannover, Germany \\ ${ }^{3}$ University of Southampton, Engineering and the Environment, Southampton, UK \\ ${ }^{4}$ Bombardier Transportation GmbH, Henningsdorf, Germany \\ 5 Technische Universität Ilmenau, Institute of Thermodynamics and Fluid Mechanics, Ilmenau, Germany \\ *Corresponding author: Keith Weinman, E-mail: keith.weinman@dlr.de
}

\section{Declarations of interest: none}

Abstract: A consistent mesh refinement study, relating to the prediction of aerodynamic forces about an experimentally validated reference train geometry, is presented in this paper. The flow about a high-speed train has a multi-scale character which poses challenges for the design of computationally effective meshes. The purpose of this study is to assist in the development of guidelines for effective drag prediction of high-speed trains using numerical simulation. These guidelines should assist CFD practitioners by identifying the regions of the mesh that are critical for the correct estimation of drag as well as providing information on appropriate mesh characteristics, such as volume and surface element length scales. Numerical assessments are validated against an experimental drag measurement program and the extent to which RANS is sufficiently predictive for industrial design is discussed. The results obtained in the work suggest that the mesh about the train nose is essential for the proper assessment of the aerodynamic drag acting on the vehicle

Keywords: aerodynamic drag, mesh refinement, mesh sensitivity, PIV

\section{Introduction}

Stringent safety requirements over a wide range of operational conditions are applied to modern high speed trains. An understanding of the aerodynamic forces acting on a vehicle is mandatory, especially under crosswind conditions, in order to construct useful operational safety constraints. The measurement of force coefficients for full-scale vehicles is optimal but expensive, and normal practices are geared towards the use of small-scale models that can be tested inexpensively in wind tunnel experiments or by using full-scale-in-service vehicles (Baker, 2010). However experimental methods are limited in scope with respect to the study of such questions as the optimization of vehicle shape over a range of design parameters. In comparison, computational methods have the potential to provide detailed flow information at a cost that is comparatively inexpensive over a much wider range of operational conditions. For example these methods can be used to determine optimal shape forms in terms of stability and drag constraints.

The use of computational methods to assess the aerodynamic loading on trains has been recognized by the transport industry. For example the German standard EN 14067-6 (DB Netz AG, 2010) permits evaluation of aerodynamic forces by means of computational fluid dynamics (CFD) simulations for full-scale or reduced model geometries. The guidelines for CFD in EN 14067-6 using RANS (Reynolds-averaged Navier-Stokes) equations are stringent and give a specific error criterion that CFD calculations must satisfy. In particular the standard requires that computed integral forces cannot be accepted for certification work if variations against accepted reference values (i.e. experiment) differ by more than three percent. A major challenge in satisfying EN 14067-6 requirements is due to the multi-scale nature of the flow problem which is characterized by a large range of energetically significant flow scales. Small-scale geometrical features of a train, for example the underflow region between the track and the train base (Sima et al., 2008), inter-car gaps and bogie cavities, can generate unsteady flow structures which interact with larger flow scales and thereby influence the development of the aerodynamic forces acting on the train. The underflow region contains numerous complex flow phenomena and is characterized typically by regions of flow separation driven by both geometry and incipient pressure gradient effects, together with cross-sectional area changes due to the underside geometry (e.g. inter-car gaps). In addition, the wake region (Muld, 2012) is dominated by vortex shedding events which contribute to the complexity of the modeling problem. Another contribution to the train aerodynamic force balance is provided by a steady vortex system originating from the front nose of the train (Baker, 2010, 2014; H. N. Hemida and S. Krajnovic, 2010). The front nose also contributes 
significantly to the train's operational drag penalty. Figure 1 presents a breakdown of contributions to the total drag budget for a full-scale train under normal operating conditions in the absence of a cross-wind. Some caution

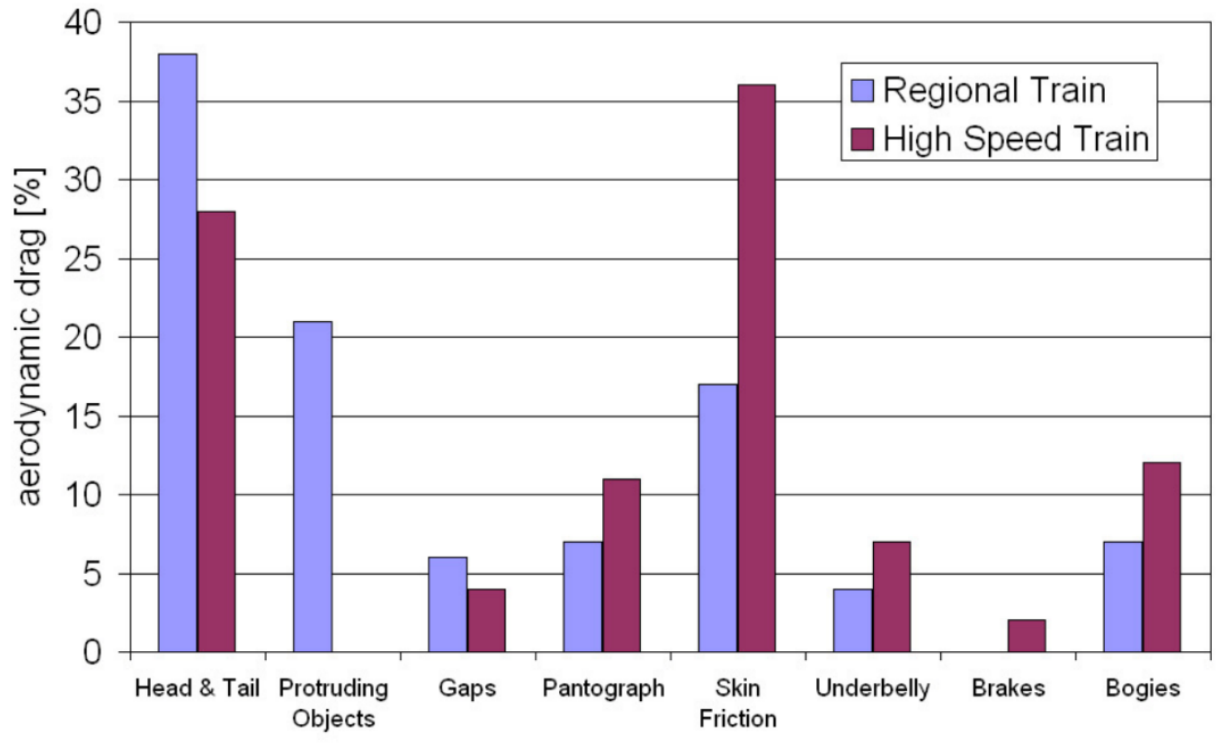

Figure 1: Contributions to the total drag of typical trains (Orellano, 2010)

must be taken in comparing this figure with scaled-model wind-tunnel data since important differences may exist, for example Reynolds number equivalence is often not possible. The figure serves, however, as a useful indicator of where the critical contributions to the operation drag budget are found. Pressure drag over the train and tail dominate. The next largest component is the total skin friction drag over the train, which can be expected to scale linearly with train length. Figure 1 implies that improvement in the prediction of head and tail drag as well as the skin friction drag will assist in the accurate assessment of operating costs for trains.

Computational assessments of the flow about a train have been traditionally undertaken on the basis of wellestablished RANS methods. The results of these examinations have not been totally satisfactory. Weinman et al. (2013) and Fragner et al. (2015) compared computational estimates of integral forces and moments using wellresolved meshes against the NGT2 experiment of Haff et al. (2012). Computations were performed for Reynolds numbers over the range $\mathcal{R} \in[250000,750000]$ with cross-wind conditions of up to 30 degrees. The computed integral force and moment coefficients, particularly drag, demonstrated differences against experimental measurements of up to 15 percent. Unsteady turbulent flow resolving methods demonstrate improved predictive capabilities over a wide range of unsteady flows when compared with RANS methods. H. N. Hemida and S. Krajnovic (2010) used Large Eddy Simulation to examine the flow over a high-speed train under cross-wind conditions. J. Morden and H.Hemida and C. Baker (2015) also investigated surface pressure loads using Delayed Detached Eddy Simulation (DDES) and a wind tunnel model. As with LES, improvements are often marginal and the efficiency of RANS methods - provided an appropriate turbulence model is available - can make it difficult to justify these computationally demanding approaches over RANS. Morden et al. obtained good results in computing vehicle surface pressure loads with RANS when using the Menter-SST model (Menter et al., 2003). Fragner et al. (2015) and Fragner and Deiterding (2016) validated highly resolved RANS, URANS, LES, Delayed Detached Eddy Simulation (DDES), and Lattice Boltzmann (LBM) methods against the NGT2 experiments. Their work demonstrated improvement for DDES, LES and the LBM methods against conventional RANS in the prediction of the aerodynamic pitching moment however differences against the measured drag were still unsatisfactory. The LBM method used returned closer agreement with experiments and demonstrated a speedup of $\sim 16$ against the competing finite volume methods, due to a novel adaptive meshing technique (Deiterding, 2011) and the explicit calculation of the LBM partial density distribution advection step. However LBM methods remain restricted at present to the low Mach number range. Unsteady methods such as LES, DDES and LBM can present challenges for use in the industrial environment. Significant computational resources are usually required. These methods have not yet demonstrated a level of improvement over RANS methods in the prediction of vehicle aerodynamic loads that would justify their use. The earlier observation of Sima et al., who noted that traditional RANS methods would retain their importance for the foreseeable future, still remains valid today. 
Investigation of the behavior of RANS methods when applied to the analysis of flows around trains is relevant for current industrial applications. A critical component of a RANS calculation is the design of the computational mesh. Mesh requirements for LES are often stated for simple flows and Spalart (2001) has provided a detailed guide for the generation of appropriate grids for hybrid RANS-LES methods such as DES. Detailed recommendations applicable in the design of RANS meshes for flows about trains appear to be absent in the literature. In this paper the influence of mesh resolution on the computed drag force of a model train is examined against experimental validation data and initial recommendations for the design of the mesh are provided. As part of this present study a series of experiments under low-speed flow conditions for a scaled model configuration were conducted using the cross-wind facility of the Simulation Center of Aerodynamic Research in Transportation (SCART) at the DLR Institute of Aerodynamics and Flow Technology located in Göttingen. This facility has been used successfully for the experimental measurements of aerodynamic forces acting on a range of ground based vehicles (Haff et al., 2012). The paper is organized as follows. Selection of the train model and experimental layout is provided in Section 2. Wind tunnel experiments are described in Section 3. An overview of the CFD geometry is given in Section 4 and Section 5 provides a description of the numerical tools used. Discussion on differences between the computed and experimental force coefficients, surface pressure distributions and wake flow structure are found in Section 6. Computational efficiency and conclusions are discussed in Sections 7 and 8, respectively.

\section{Train model and experimental setup}

For this paper a model consisting only of the main train aerodynamic surfaces and the wind tunnel is considered. Additional geometrical features, such as inter-car gaps and bogie cavities, are not considered in the present investigation: the goal is to examine the influence of mesh resolution using the simplest representative geometry. Figure 2 illustrates the wind tunnel model as well as the coordinate system used for both the experiment and numerical calculation.

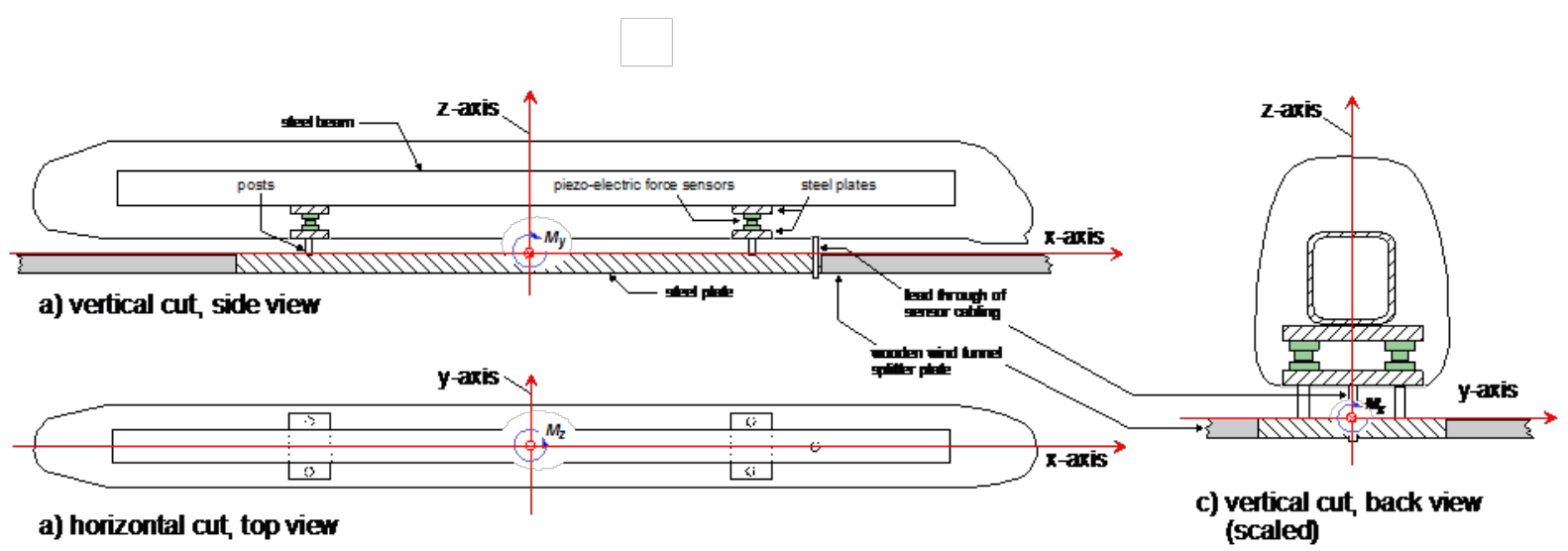

Figure 2: Sketch of the experimental setup.

The reference system is aligned with the stationary inertial reference frame of the wind tunnel. Further details of the model can be seen in Figure 2. The model segments are fastened to a hollow steel $0.1 \times 0.1 \mathrm{~m}^{2}$ beam. Two Kistler Piezo-electric sensors of type 9317-B are mounted between two $0.02 \mathrm{~m}$ thick steel plates, and two of these assemblies are located with a separation of $1.2 \mathrm{~m}$ on the steel beam. The lower parts of the assemblies are fixed to a $1.6 \mathrm{~m} \mathrm{x} 0.2 \mathrm{~m}$ steel plate of $0.02 \mathrm{~m}$ thickness, which is embedded into the wooden wind tunnel splitter plate (3.302 $\mathrm{m}$ in length), using posts (see Figure 2). The plate extends from the left tunnel wall to the right tunnel wall. The mono-block model consists of two end cars connected via the steel beam at 1/15 scale of a full-sized vehicle. The model scale was chosen on the basis of a numerical study on the wind tunnel blocking effect as a function of the model scale (Fragner, 2015). A Pencil model head geometry is used (Figure 3a), while a trailing Crespin head (Figure 3b) is fixed to the rear of the model. The model train height $\left(L_{h}\right)$ is $0.25 \mathrm{~m}$ with the train base having a width $\left(L_{b}\right)$ of $0.16 \mathrm{~m}$. Specific details of the geometry are confidential but note that the bounding box enclosing the train geometry is given in Section 6.2, Table 5. The definition of the characteristic length for the Reynolds number within the industrial train research community is motivated by difficulties in comparing different vehicle configurations. For example, the height of single- and double-deck vehicles can vary significantly but the widths are approximately constant due to the standard rail gauge the vehicles must operate on. A reference width of $3 \mathrm{~m}$ at full scale is commonly used in Europe. The scaled reference width provides the reference length used to compute the Reynolds number for the wind tunnel experiment. The Reynolds number is defined as Eq. (1), 


$$
\mathcal{R}=\rho L U / \mu,
$$

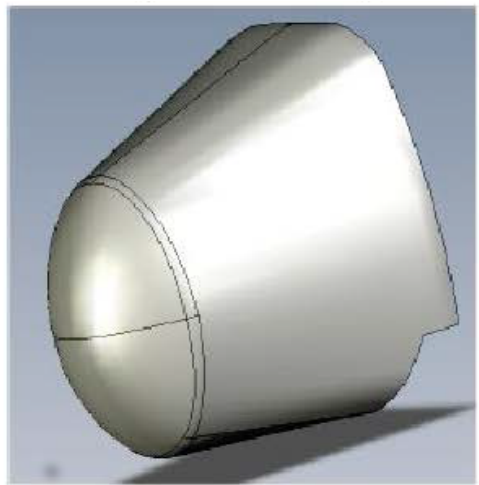

(a) Pencil head (streamlined)

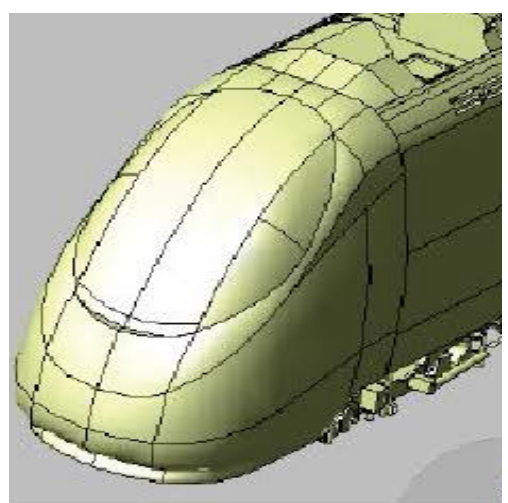

(b) Crespin head

Figure 3: Leading and trailing model train head configurations

\section{Wind Tunnel Facility}

Experiments under low-speed flow conditions were conducted in the cross-wind facility Seitenwindkanal Göttingen (SWG) located in the Simulation Center of Aerodynamic Research in Transportation (SCART) in the Institute of Aerodynamics and Flow Technology at the DLR Göttingen. This facility is illustrated in Figure 4.

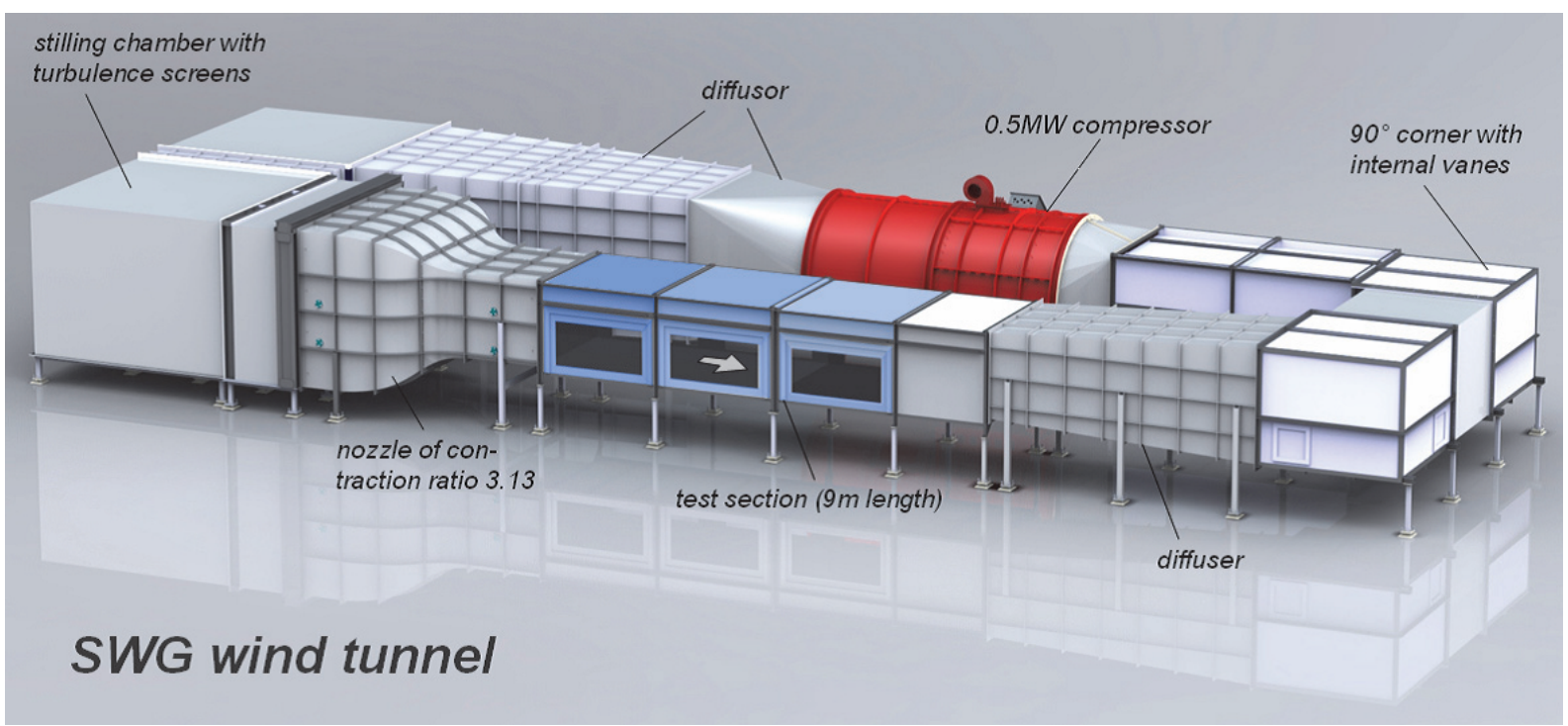

Figure 4: 3D design image of the cross-wind facility (SWG) of DLR Göttingen

The SWG is equipped with a $9 \mathrm{~m}$ long test section with a cross section of $2.4 \mathrm{~m}$ (width) $\mathrm{x} 1.6 \mathrm{~m}$ (height). The wind tunnel is a continuously working, atmospheric Göttingen-type wind tunnel with a 3.13 contraction driven by a $0.5 \mathrm{MW}$ compressor. It can be operated in the flow-speed range $2<U<65 \mathrm{~m} / \mathrm{s}$ and the maximum Mach number that can be achieved in the SWG is $\sim 0.21$. Wind tunnel measurements were performed at the bulk flow parameters given in Table 1. 


\begin{tabular}{|c|c|c|c|c|c|}
\hline$U(\mathrm{~m} / \mathrm{s})$ & $T(\mathrm{~K})$ & $\mathrm{P}(\mathrm{hPa})$ & $\mathcal{R}$ & Mach Number & $\nu\left(\mathrm{m}^{2} / \mathrm{s}\right)$ \\
\hline 61.6 & 300 & 1013 & $9.4226 \mathrm{e}+05$ & 0.177 & $1.568 \mathrm{e}-05$ \\
\hline
\end{tabular}

Table 1: Bulk flow parameters at tunnel conditions. Note $\nu$ is the kinematic viscosity.

The model is mounted on a splitter plate located $0.2 \mathrm{~m}$ above the lower tunnel wall. The distance from the model train nose to the upstream edge of the splitter plate is $1.8 \mathrm{~m}\left(11.25 \mathrm{~L}_{b}\right)$. Figure 5 illustrates the locations of Pitot-static tubes $\left(U_{1}, U_{3}\right)$ used for dynamic pressure measurements as well as the locations of Laser Doppler Anemometry cuts $\left(L_{1}, L_{5}\right)$ which traverse normal to the upper surface of the splitter plate.

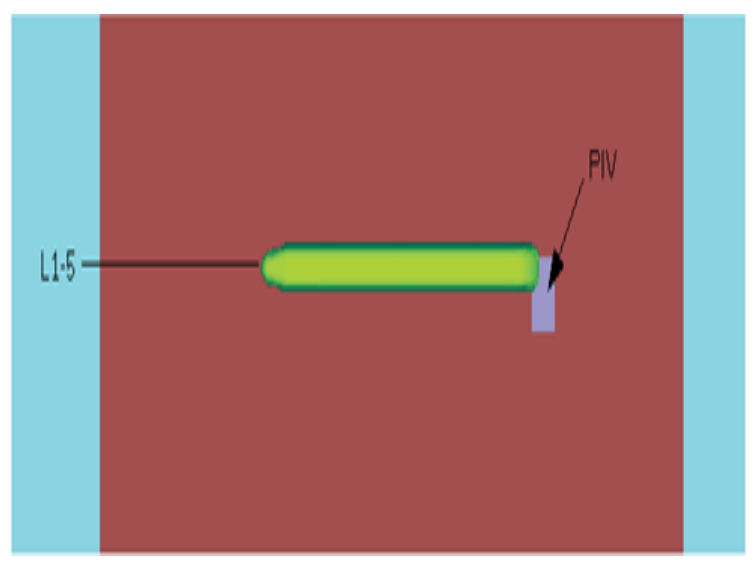

(a) View in the x-y plane.

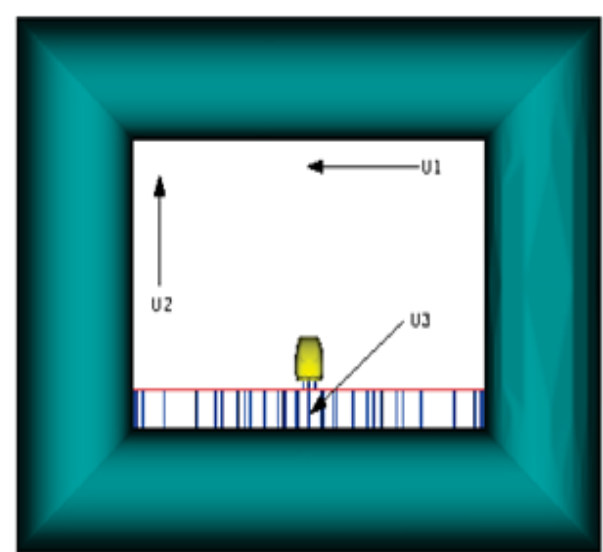

(b) View in the y-z plane.

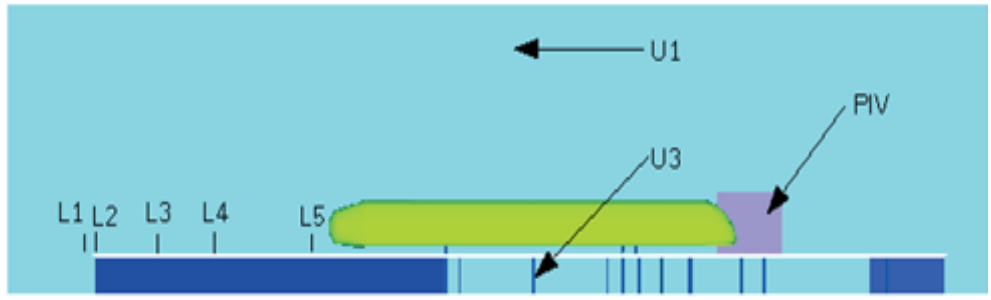

(c) View in the $x-z$-plane

Figure 5: Locations of sensor positions relative to the model geometry. Note that the LDA line cut positions are shown at positions $\left(L_{1}, \cdots, L_{5}\right)$. Three $y$ positions $( \pm 0.5,0.0) \mathrm{m}$, together with five $x$ positions relative to the plate leading edge $(-0.035,0,0.400,0.80,0.145) \mathrm{m}$ are used. Only the LDA position at $y=0$ is shown in the figures. The Pitot-static tube locations within the tunnel reference frame are normalized with $L_{b}$ here and are given by: $x_{U_{1}}=(0.37375,0,7.1875)(0.25$ $\mathrm{m}$ down from the upper tunnel wall), $x_{U_{2}}=(0,6.90625,6.4375)(0.37 \mathrm{~m}$ down from upper wall and $0.255 \mathrm{~m}$ right of the left side wall), $x_{U_{3}}=(1.65625,0,-0.64062)(0.0975 \mathrm{~m}$ up from the lower tunnel wall).

The turbulence intensity levels in the mean flow direction, determined with hot-wire anemometry across the vertical centerline of the test section of the empty wind tunnel, were measured to be 0.12 percent at a bulk velocity at position $U_{1}$ of $20 \mathrm{~m} / \mathrm{s}$ and 0.24 percent for $60 \mathrm{~m} / \mathrm{s}$. These values satisfy EN 14067-6 specifications. Positions of the pitot static tubes are given in the caption of Figure 5. Particle Image Velocimetry (PIV) was used to obtain velocity vectors of the averaged flow fields in the horizontal $(x-y)$ and vertical $(x-z)$ planes. These are shown in closer detail in Figure 6a and Figure 6b. A Nd-Yag laser with a pulse frequency of $10 \mathrm{~Hz}$ was used to generate the laser light sheet. Time shifts of 30 and $50 \mu \mathrm{s}$, based on the comparative sizes of the PIV cuts, were selected for the horizontal and vertical light sheets respectively. Di-Ethyl-Hexyl-Sebacat droplets, with a mean diameter of $1 \mu \mathrm{m}$, were used as tracer particles and images were recorded using a PCO 2000 CCD camera with a resolution of $2048 \times 2048$ pixels. Collection of the PIV data was performed over approximately 600 time frames, corresponding to a period of 70 seconds in physical time. Averages were assessed with 315 images for each run. Note that initial and final images were discarded. The intermediate slices were taken at intervals to reduce correlation effects between successive samples. Figure A.1 in Appendix A illustrates the variations in density and the $x$-velocity component for a typical single force measurement run at the operating conditions given in Table 1. Mean conditions vary slightly over the duration of the test period. The measuring time for data acquisition at higher Mach 
numbers is limited since the SWG is not equipped with a heat exchanger. Therefore the wind tunnel flow bulk temperature increases at maximum through-flow conditions. As the fluid bulk temperature rises both the density and fluid viscosity decrease. A control system adapts the bulk fluid velocity to maintain the Reynolds number to within a set point deviation of less than $\pm 0.5 \%$. The force and moment measurements that are performed using this method are highly reproducible. Note that the experiments have been optimized for the collection of aerodynamic drag.

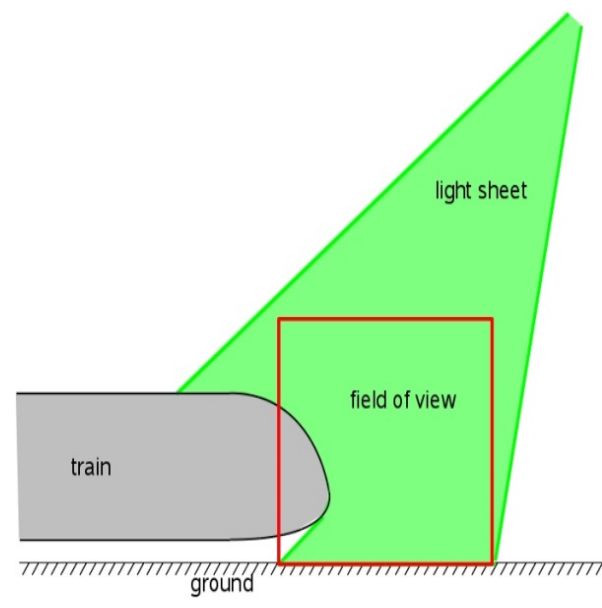

(a) Plane S1 given by $(x,-0.05, z) \mathrm{m}$

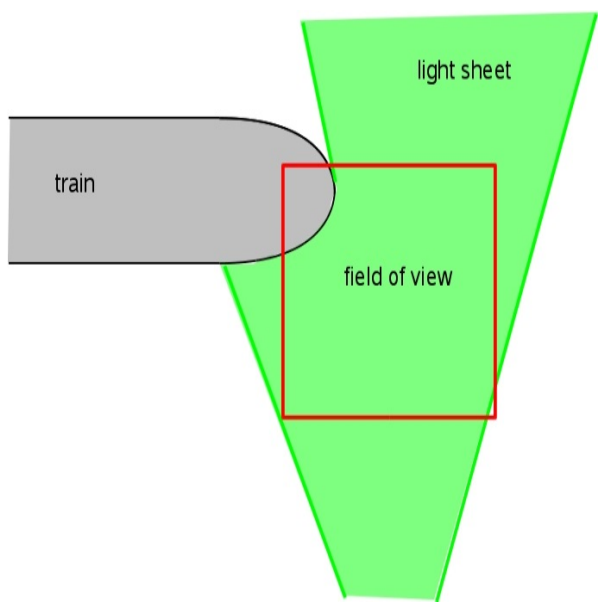

(b) Plane $\mathrm{S} 2$ given by $(x, y, 0.12) \mathrm{m}$

Figure 6: PIV light sheet definitions.

\section{Computational domain, CFD model and Turbulence Model}

Figure 7 illustrates the CFD model which includes the model, splitter plate and the SWG wind tunnel. Dimensions of the wind tunnel, plate, and refinement zones are given in Sections 2, 3 and Table 5 respectively.

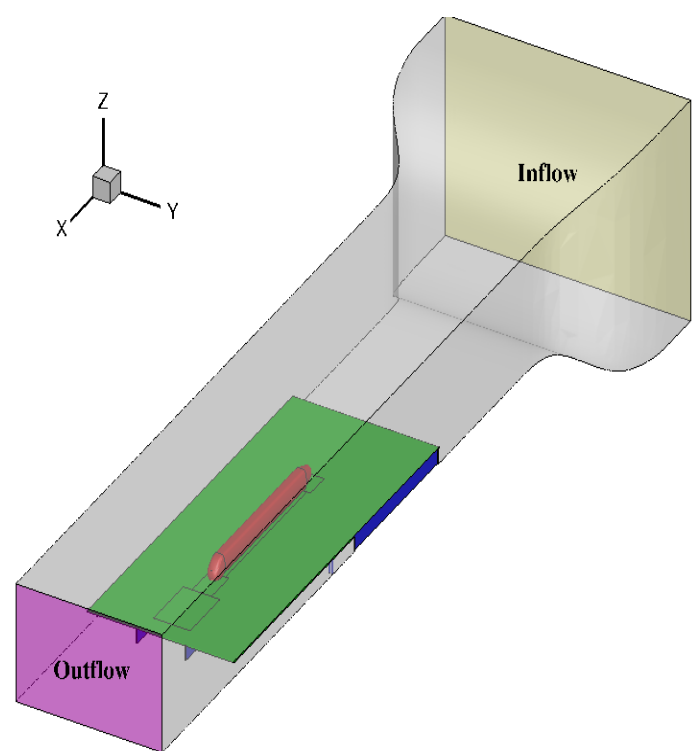

(a) Full domain.

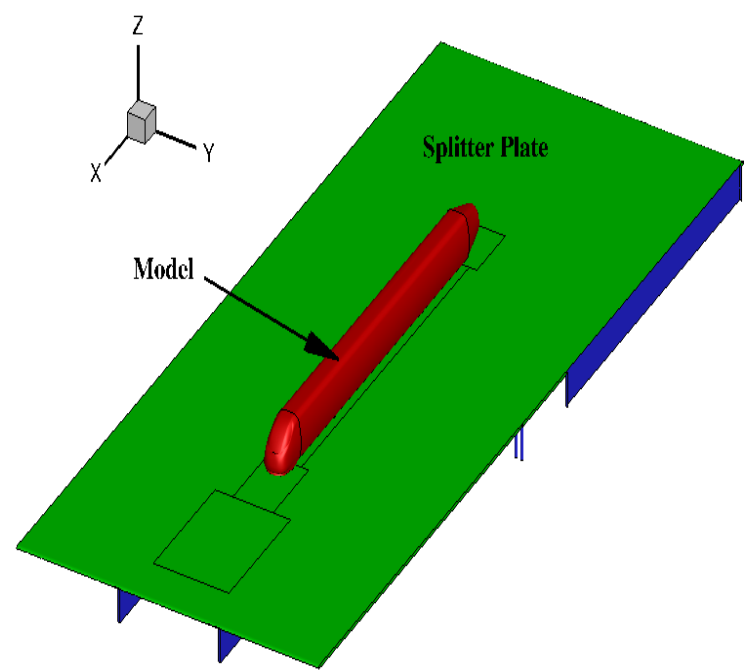

(b) View of model on Splitter Plate

Figure 7: The computational domain.

Locations of the domain inflow and outflow surfaces are shown in Figure 7(a). All other surfaces are defined as no-slip walls with their boundary conditions given in Table 2 in Section 5. The boundaries of the refinement regions, projected in the $z$-direction, are indicated on the splitter plate in Figure 7(b). The CFD model includes all 
mounting posts and mounting rails located underneath the splitter plate.

\title{
5 The Numerical Method
}

The solution of all equations used in the mathematical description of the flow was undertaken with the OpenFOAM library (Weller et al., 1998). Further details of the numerical solver used are provided in Appendix C. The incompressible form of the momentum and continuity equations, which form the basis for the mathematical description of the fluid flow, are written below:

$$
\begin{aligned}
\partial_{t} u+u \cdot \nabla u & =-\frac{1}{\rho} \nabla p+\nu \nabla^{2} u, \\
\nabla \cdot u & =0 .
\end{aligned}
$$

Justification for the choice of the incompressible form of the Navier-Stokes equations is given in Appendix A. Turbulence effects are conventionally modeled by replacing the dynamic viscosity $\nu$ in Equation (2) with an effective viscosity being given by $\nu^{*}=\nu+\nu_{T}$ (Wilcox, 2006). The turbulence model used to provide this closure is described in Appendix B. Boundary conditions are shown in Table 2.

\begin{tabular}{|c|c|c|c|c|}
\hline & $P$ & $U$ & $k$ & $\omega$ \\
\hline Inflow & $\nabla P \cdot n=0$ & $U=U_{\text {in }}$ & $k=k_{\text {in }}$ & $\omega=\omega_{\text {in }}$ \\
\hline Outflow & $P=P_{\infty}$ & $\nabla U \cdot n=0$ & $\nabla k \cdot n=0$ & $\nabla \omega \cdot n=0$ \\
\hline Non-slip walls & $\nabla P \cdot n=0$ & $U=0$ & $k=0$ & $\omega=\omega_{\text {wall }}$ \\
\hline
\end{tabular}

Table 2: Boundary conditions - note that $\mathrm{n}$ is the unit face normal vector

\begin{abstract}
Values of $\omega$ and $\mu_{T}$ at the wall used are based on a proposal by Menter et al. (2003). A blending function, based on the dimensionless wall distance $y^{+}$, acts as an automatic wall treatment in order to minimize deterioration of wall model predictive abilities when a low Reynolds model is applied on an under-resolved grid. For inflow boundaries the turbulent eddy viscosity is computed as $\mu_{T}=k / \omega$, while a zero gradient condition is employed at the outflow. Table 3 provides data on the length of the data used for averaging. Integral data was stored for each iteration. Ensemble averaging was used to then determine when stationary values of the average force coefficients were returned. Equations were integrated from this point for an additional 10000 iterations in order to estimate average force coefficients. The table shows some variations in the number of iterations required for initialization due to unsteadiness in the underflow region and wake regions.
\end{abstract}

\begin{tabular}{|c|c|c|c|c|c|c|c|}
\hline \multirow{2}{*}{ Period } & \multicolumn{2}{|c|}{ Front nose } & \multicolumn{2}{c|}{ Underfloor } & \multicolumn{2}{c|}{ Wake } & Reference \\
\cline { 2 - 8 } & $M_{1}^{F N}$ & $M_{3}^{F N}$ & $M_{1}^{U F}$ & $M_{3}^{U F}$ & $M_{1}^{W}$ & $M_{3}^{W}$ & $M^{r e f}$ \\
\hline Initialization & 3896 & 5112 & 4116 & 4766 & 5156 & 4050 & 5323 \\
\hline Averaging & 10000 & 10000 & 8000 & 5000 & 10000 & 10000 & 10000 \\
\hline
\end{tabular}

Table 3: A comparison of the number of iterations used for initialization and for averaging.

CFD results are compared against experiment in this section. Integral forces are compared and an assessment on the best possible refinement strategy for agreement against the experimentally measured drag coefficient is provided. A brief discussion on the influence of the mesh refinement strategies on the computed surface pressure profiles is then provided. Finally PIV data in the wake are compared against the CFD.

\subsection{Validation of the CFD method}

The inflow tunnel conditions for the CFD were assessed at the design Reynolds number, given in Table 1, using CFD. Computed and experimental velocity magnitudes at three pitot tube positions were compared. Table 4 
contains the mean values of the measured velocity magnitude at the pitot tube positions $U_{1, . .3}$ together with the percentage variation of the computed magnitudes against the experiment at these positions, which is given by

$$
E_{\phi}=10^{2} * \frac{\left(\phi^{\text {numerics }}-\phi^{\text {experiment }}\right)}{\phi} .
$$

Here $\phi$ is the variable of interest (for this table $\phi$ is the averaged velocity magnitude $\bar{U}$ ). The experimental results were averaged over four separate tests runs, with the data collection for each time being of the order of several hundred flow integral time scales. The variation of $\mathrm{O}(3.5 \%)$ above the splitter is considered acceptable. This variation increases to $\mathrm{O}(12.6 \%)$ for the probe $U_{3}$, which is located under the splitter plate. This is due to the highly unsteady velocity field induced by the support pylons for which a sufficiently fine mesh would be required to properly resolve essential under-plate flow physics. Additional resolution is not required here as the splitter plate effectively shields the model and the flow above the plate from this region.

\begin{tabular}{|c|c|c|c|}
\hline & $\overline{U_{1}}(\mathrm{~m} / \mathrm{s})$ & $\overline{U_{1}}(\mathrm{~m} / \mathrm{s})$ & $\overline{U_{1}}(\mathrm{~m} / \mathrm{s})$ \\
\hline Experiment & $61.6 \pm 0.25$ & $61.4 \pm 0.25$ & $51.6 \pm 0.21$ \\
\hline \multicolumn{4}{|c|}{ Percentage error : Eq. 4} \\
\hline $\mathrm{E}(\bar{U})$ & -3.571 & -3.5831 & 12.597 \\
\hline
\end{tabular}

Table 4: Comparison of experimental and computed mean velocity magnitudes at static probe positions.

\begin{abstract}
A zero pressure gradient flat plate problem was then used to validate both the choice of near-wall discretization mesh parameters and turbulence model. Of interest is the influence of near-wall gridding and near-wall turbulence model performance on predicting skin friction accurately. Two cases, at Reynolds numbers of 0.63 and 6.3 Million were considered (the Reynolds number is defined using Eq. (1) with $L_{b}$ being replaced by the plate length) and compared against analytical profiles of skin friction, velocity and turbulence parameters. For the Menter-SST (Menter, 1994) turbulence model typical deviations in the profiles of predicted skin friction against published skin friction profiles for flat plates (with no roughness) were in the order of 2 percent up to a dimensionless wall distance (rescaled by viscous units) of $y^{+}<100$ (Fragner, 2015).
\end{abstract}

\subsection{Mesh Refinement Studies}

In this section the solutions returned by using identical numerics on sequences of refined meshes are compared and discussed at conditions given by Table 1 . The general strategy chosen is to examine the effects of both a consistent refinement and derefinement of a reference mesh, selected by numerical experiment, on the force coefficients predicted by the numerical scheme. Mesh refinement regions are selected on the specifications of Baker (Baker, 2010):

- front nose $(\mathrm{FN})$ region,

- underflow (UF) region,

- near and far wake $(\mathrm{W})$ regions,

- and attached boundary layers.

The bounding box coordinates of the spatial volumes occupied by the model train and the refinement regions, defined within the coordinate system described in Figure 2, are given in Table 5. 


\begin{tabular}{|c|c|c|c|c|c|c|}
\hline Geometry & $x_{1} / L_{b}$ & $x_{2} / L_{b}$ & $y_{1} / L_{b}$ & $y_{2} / L_{b}$ & $z_{1} / L_{b}$ & $z_{2} / L_{b}$ \\
\hline Model train & -8.77019 & 8.71031 & -0.61594 & 0.61594 & -0.12125 & 1.72011 \\
\hline FN & -9.68750 & -7.2285 & -1.0 & 1.0 & -0.12125 & 1.8125 \\
\hline UF & -7.2285 & 7.2285 & -0.625 & 0.625 & -0.12125 & 0.135 \\
\hline NW & 7.2285 & 10.312 & -1.0 & 1.0 & -0.12125 & 1.8125 \\
\hline FW & 10.312 & 15.625 & -1.875 & 1.875 & -0.12125 & 1.8125 \\
\hline
\end{tabular}

Table 5: Bounding box dimensions normalized on the model width $L_{b}$.

In the following sections $M_{c}^{r}$ is used to symbolically denote a refinement level $c$ performed on a region $r$ of the mesh $M$. The values of $c$ are given by $c=[-1,0,1]$, representing a single level of derefinement, no refinement, and a single level of refinement respectively. No refinement/derefinment for any region $r$ exists in the reference mesh $(c=0)$, and $M_{0}$ symbolically represents the reference mesh. For example, $M_{-1}^{F N}$ denotes the reference mesh with a (single) derefinement in the front nose region. We define an operator $L\left(M_{c}^{r}\right)$ which returns the spatially averaged length scale of the mesh region $M^{r}$ at some refinement level $c$. The following constraint for $L\left(M_{c}^{r}\right)$ is true for all refinements performed in this work:

$$
L\left(M_{0}\right) / L\left(M_{-1}^{r}\right) \approx L\left(M_{1}^{r}\right) / L\left(M_{0}\right) \approx 2.0
$$

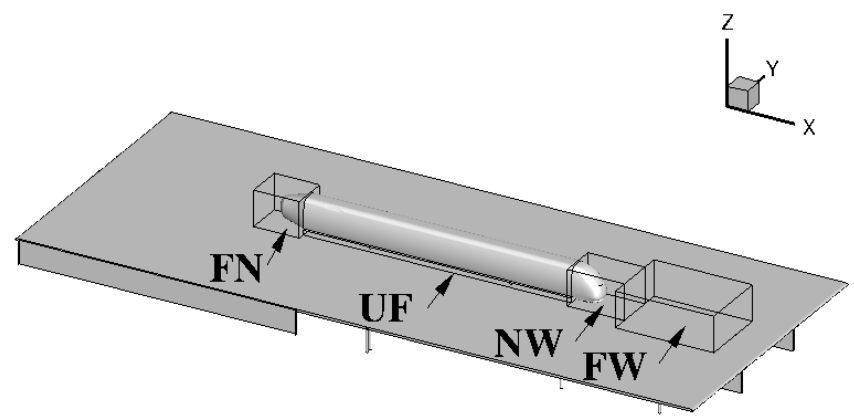

Figure 8: Refinement regions.

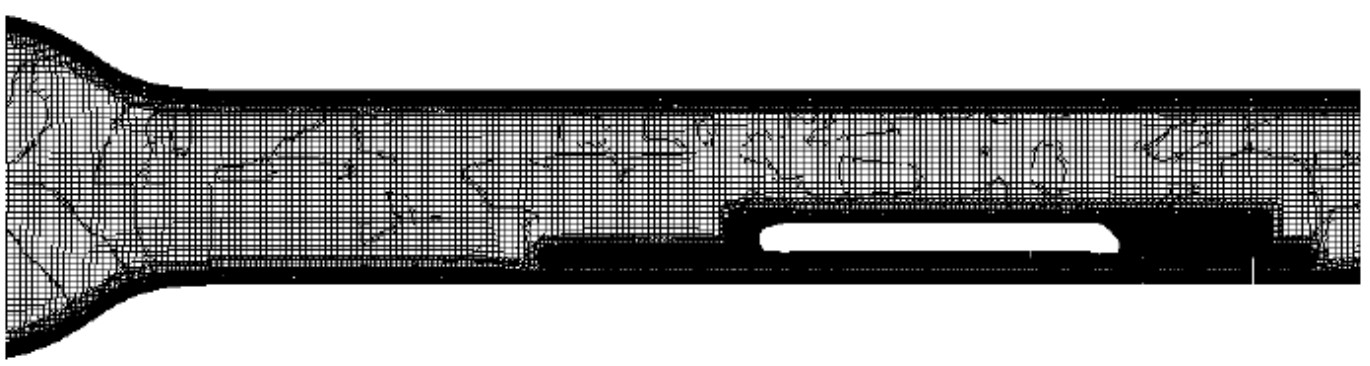

Figure 9: Refinement regions and model embedded in background mesh.

Figure 8 illustrates the refinement regions used. A perspective of the refinement regions embedded into the reference mesh is shown in Figure 9. Details of $y^{+}$and surface element length scales in the refinement regions are provided in Table 6. External to the refinement regions and walls, the embedding mesh away from walls consists of uniform hexahedral elements with a length scale of approximately $L_{b} / 5$. 


\begin{tabular}{|c|c|c|c|c|c|c|c|}
\hline \multirow{2}{*}{ Mesh } & Front nose & \multicolumn{2}{c|}{ Underfloor } & \multicolumn{2}{|c|}{ Wake } & Reference \\
\cline { 2 - 8 } & $M_{-1}^{F N}$ & $M_{1}^{F N}$ & $M_{-1}^{U F}$ & $M_{1}^{U F}$ & $M_{-1}^{W}$ & $M_{1}^{W}$ & $M_{0}$ \\
\hline \multicolumn{7}{|c|}{ Train $y^{+}$values } \\
\hline Front nose & 43 & 45 & 45 & 45 & 45 & 45 & 45 \\
\hline Underflow & 27 & 33 & 27 & 33 & 33 & 33 & 33 \\
\hline Back nose & 33 & 33 & 33 & 33 & 33 & 34 & 34 \\
\hline Train & 35 & 35 & 35 & 34 & 35 & 34 & 34 \\
\hline Posts & 30 & 29 & 29 & 32 & 31 & 31 & 31 \\
\hline \multicolumn{7}{|c|}{ Plate $y^{+}$values } \\
\hline Front nose & 32 & 33 & 34 & 34 & 34 & 34 & 34 \\
\hline Underflow & 33 & 33 & 27 & 33 & 32 & 32 & 32 \\
\hline Near Wake & 30 & 36 & 30 & 32 & 28 & 31 & 31 \\
\hline Far Wake & 36 & 36 & 36 & 36 & 36 & 36 & 36 \\
\hline Remaining & 115 & 113 & 118 & 117 & 116 & 115 & 115 \\
\hline \multicolumn{7}{|c|}{ Surface element length scales (mm) } \\
\hline Front Nose & 46.8 & 11.7 & 23.4 & 23.4 & 23.4 & 23.4 & 23.4 \\
\hline Underflow & 23.4 & 23.4 & 46.8 & 11.7 & 23.4 & 23.4 & 23.4 \\
\hline Near Wake & 23.4 & 23.4 & 23.4 & 23.4 & 46.8 & 11.7 & 23.4 \\
\hline Far Wake & 46.8 & 46.8 & 46.8 & 46.8 & 93.7 & 23.4 & 46.8 \\
\hline
\end{tabular}

Table 6: Details of meshes used in the refinement study

The Open-FOAM tool, SnappyHexMesh, was used for mesh generation. The distribution of near-wall cells in the wall normal direction is uniquely specified by the number of layers, the expansion ratio (ratio of wall-normal length scales between successive control volumes) and the final or first layer thickness (HELYX, 2014). Values of $y^{+}$in the range $y^{+} \in[29,45]$, validated for the chosen turbulence model using the zero pressure gradient boundary layer problem discussed in Section 6.1, were selected. The required range of $y^{+}$values were returned with a combination of the final layer thickness at a fraction of 0.8 of the adjacent surface mesh size, an expansion ratio of 1.25, and with the number of wall layers varying from 5 to 8 . Wall-normal control volume distributions were held fixed in the near-wall region for all refinements. Surface elements are modified as part of the refinement in directions tangent to the underlying surface. Table 6 illustrates that the variations in $y^{+}$values across the refinements performed are negligible. Note that near-wall resolution requirements for the hybrid wall function approach used (Section 5) do not require $y^{+} \leq O(1)$ as do low Reynolds number wall modeling approaches (Wilcox, 2006). Small variations in the fluid near-wall stresses are seen in the table, but these variations do not appear to play a significant role in the computed aerodynamic loads. Appendix D provides information on refinement levels and other relevant parameter settings used for the reference mesh generation. All meshes are compliant with the requirements of the EN-1407-6 standard. The constraint, given by Eq. (5), is satisfied across all refinements. Mesh length scales and $y^{+}$values outside of the refinement regions are sufficiently fine to predict the mean flow and are not discussed further in this work. Properties of $M_{0}$ can be deduced from the settings given in Appendix D.

This work is primarily concerned with the prediction of drag. Lift is only of secondary importance for this paper. As noted in Section 3, the experiments are optimized for drag measurements only. Drag and lift coefficients are defined below by Eq. 6 and Eq. 7 respectively. Here $D$ is the drag force, $L$ is the lift force, $q_{\infty}$ is the dynamic pressure and $S$ is the reference area (Anderson and Tannehill, 1984).

$$
\begin{aligned}
C_{d} & =\frac{D}{q_{\infty} S} \\
C_{l} & =\frac{L}{q_{\infty} S}
\end{aligned}
$$


Differences between computed and measured drag coefficients are presented in terms of lift and drag count differences against experiment, as well as the percentage error $E$ given by (4),

$$
\begin{aligned}
& L\left(C_{d}\right)=10^{4}\left(C_{d}^{\text {numerics }}-C_{d}^{\text {experiment }}\right) \\
& L\left(C_{l}\right)=10^{2}\left(C_{l}^{\text {numerics }}-C_{l}^{\text {experiment }}\right)
\end{aligned}
$$

whereby, from the above equations, it is clear that one drag count is equivalent to $\triangle C_{d}=0.0001$ and one lift count is equal to $\triangle C_{l}=0.01$. The left-hand sides of equations (8) and (9) represent the differences between measured and computed values. The ratio of the viscous drag force to the total aerodynamic drag force is given by

$$
R=\frac{C_{d}^{\nu}}{C_{d}^{p}+C_{d}^{\nu}}
$$

$R$ provides a metric for comparison against Figure 1. Results for the integral force analysis are shown in Table 7. Note that absolute values of the difference terms are not used: interest is in convergence towards an asymptotic limit. The value of $R$ shown in the table is in correspondence with the values estimated in Figure 1, with most calculations returning a fraction of about 0.35. The fraction returned for the finest nose refinement is about 0.4 due to the improved drag estimate. EN 14067-6 permits an upper limit of 3 percent for the variation between experimentally and numerically assessed drag values. The nose refinement strategy provides drag coefficients which demonstrate an approach to an asymptotic limit over a sequence of three successive refinements with $M_{1}^{F N}$ and returns an estimate which satisfies the EN-14067-6 requirement. The refinement in the near and far wake regions

\begin{tabular}{|c|c|c|c|c|c|c|c|c|c|c|}
\hline Mesh & $-C_{d}$ & $\left(E_{C_{d}}\right) \%$ & $L\left(C_{d}\right)$ & $-C_{l}$ & $\left(E_{C_{l}}\right) \%$ & $L\left(C_{l}\right)$ & $R$ & $-C_{d}^{p}$ & $-C_{l}^{\nu}$ & $-C_{l}^{p}$ \\
\hline \multicolumn{11}{|c|}{ Reference mesh } \\
\hline$M_{0}$ & 0.3759 & 11.1 & 376 & 0.245 & 64.4 & 10 & 0.351 & 0.244 & 0.001 & 0.244 \\
\hline \multicolumn{11}{|c|}{ Near/far wake refinement } \\
\hline$M_{-1}^{W}$ & 0.3717 & 9.9 & 334 & 0.253 & 69.8 & 11 & 0.357 & 0.239 & 0.001 & 0.252 \\
\hline$M_{1}^{W}$ & 0.3745 & 10.7 & 362 & 0.245 & 64.4 & 10 & 0.345 & 0.245 & 0.001 & 0.244 \\
\hline \multicolumn{11}{|c|}{ Underflow refinement } \\
\hline$M_{-1}^{U F}$ & 0.3225 & -4.7 & -158 & 0.197 & 32.2 & 5 & 0.349 & 0.192 & 0.001 & 0.196 \\
\hline$M_{1}^{U F}$ & 0.3571 & 5.6 & 188 & 0.227 & 52.3 & 8 & 0.353 & 0.224 & 0.001 & 0.226 \\
\hline \multicolumn{11}{|c|}{ Front nose refinement } \\
\hline$M_{-1}^{F N}$ & 0.3806 & 12.5 & 423 & 0.247 & 65.7 & 10 & 0.347 & 0.248 & 0.001 & 0.246 \\
\hline$M_{1}^{F N}$ & 0.3351 & -0.9 & -32 & 0.202 & 35.6 & 6 & 0.397 & 0.202 & 0.001 & 0.201 \\
\hline \multicolumn{11}{|c|}{ Front nose and underflow refinement } \\
\hline$M_{-1}^{F N+U F}$ & 0.3079 & -8.9 & -301 & 0.186 & 24.8 & 4 & 0.418 & 0.179 & 0.001 & 0.185 \\
\hline$M_{1}^{F N+U F}$ & 0.3301 & -2.4 & -82 & 0.204 & 36.2 & 5 & 0.390 & 0.201 & 0.001 & 0.203 \\
\hline \multicolumn{11}{|c|}{ Experiment } \\
\hline Exp. & $0.3383 \pm 0.009$ & - & - & $0.149 \pm 0.001$ & - & - & - & - & - & - \\
\hline
\end{tabular}
return errors against the experiment of 10 percent (357 drag counts) on average, and no asymptotic convergence to a limit is demonstrated.

Table 7: Comparison of force coefficients

Differences between the computed and experimental drag increase and then decrease as the underflow region is refined. An error of 5.6 percent (188 counts) is returned on the finest mesh. This error is of the same order as the magnitude of error computed on the coarsest mesh. The error magnitude is smaller in comparison to that of the 
wake refinements but the EN-14067-6 requirement is not met. A smooth approach to a limit is not demonstrated, and convergence may be oscillatory. Additional refinement studies could be performed in this region however bounds on the error magnitude do not change significantly. Given the focus of the work, it is difficult to justify a case for further refinement of the reference mesh for a RANS method in the underflow and wake regions since these regions appear to be non-critical for model drag estimation. While this suggests that the refinement criterion for these two regions is possibly more demanding than that achieved in this work, the underflow and the wake regions contain a range of challenging flow phenomena (e.g. streamline curvature, adverse pressure gradients) for which weaknesses of the RANS approach are well documented (Wilcox, 2006; W. Haase and M.Braza and A. Revell, 2009). The goal of this paper is to identify mesh regions which are critical for drag prediction and results obtained in this work suggests that accurate prediction of the wake and underfloor regions may not be critical if the front nose flow is sufficiently well resolved. Table 8 presents guidelines for the wall normal resolution and mesh length scales for the refinement regions which are deduced from Table 6 . These guidelines should be formally relevant for RANS linear eddy viscosity models at or near the Reynolds number used in this work.

\begin{tabular}{|c|c|c|}
\hline Refinement Region & $y^{+}$ & $L / L_{b}$ \\
\hline Near Wake & 28 & 0.1 \\
\hline Far Wake & 55 & 0.21 \\
\hline Underflow & 33 & 0.1 \\
\hline Nose & 45 & 0.026 \\
\hline
\end{tabular}

Table 8: Values of $y^{+}$and mesh length scales recommended for the test geometry at the Reynolds number given in Table 1.

We follow the recommendations of Celik et al.(I. B. Celik and U. Ghia and P. J. Roache and C. J. Freitas and H. Coleman and P. E. Raad, 2008) in estimating the numerical error of our calculations. The estimates are based on a refinement of the Richardson extrapolation technique (L.F. Richardson, 1910), the Grid Convergence Index method (GCI) (P. J. Roache, 1998). Mesh refinements in this work are not global, but are embedded locally within the mesh. Furthermore, refinements are not applied uniformly to the regions abutting the drag inducing surfaces. This makes application and interpretation of a method based on a global refinement difficult. Since our principal concern is in the prediction of integral forces, defined on the model surface, we start by estimating a representative surface mesh size. The observation that surface viscous stress contributions and the farfield solutions upstream of the wake do not demonstrate an obvious dependence on the refinements preformed suggests a representative length scale $h$ based on the average size of the model's surface mesh length scales, which can be given as

$$
h=\sqrt{\frac{1}{N} \sum_{i=1}^{N}\left(\triangle A_{i}\right)},
$$

where $N$ is the total number of surface elements and $\triangle A_{i}$ is the $i^{\text {th }}$ surface area element. The grid refinement is local to regions of the model surface and we look at GCI estimates based on the refined front nose region. This implies that we can use $h \approx \sqrt{1 / N}$, where $N$ is the number of faces covering the front nose region surface. Note that the surface faces are reasonably isotropic (verified by inspection and by inspection of the length scale ratios across refinements given in Table 9). Celik et al. suggests that the grid refinement factor $r=h_{\text {coarse }} / h_{\text {fine }}$ should be larger than 1.3. Three refinements are first chosen such that $h_{1}<h_{2}<h_{3}$ with $r_{21}=h_{2} / h_{1}$ and $r_{32}=h_{3} / h_{2}$. The variable of interest estimated on each of these grids are given by $\phi_{i}, \phi_{2}$ and $\phi_{3}$. The change in $\phi$ for each refinement is given by $\epsilon_{21}=\phi_{2}-\phi_{1}$ and $\epsilon_{32}=\phi_{3}-\phi_{2}$. Following Celik et al., fix-point iteration can be used to solve the following system to estimate the order of convergence $p$.

$$
\begin{aligned}
p & =\frac{1}{\ln \left(r_{21}\right)}|\ln | \epsilon_{32} / \epsilon_{21}||+q(p) \\
q(p) & =\ln \left(\frac{r_{21}^{p}-s}{r_{3}^{p} 2-s}\right) \\
s & =1-\operatorname{sgn}\left(\epsilon_{32} / \epsilon_{21}\right)
\end{aligned}
$$

An estimate of the exact solution $\phi_{\text {ext }}$, extrapolated from the fine grid solution, is given by

$$
\phi_{\text {ext }}=\left(r_{21}^{p} \phi_{1}-\phi_{2}\right) /\left(r_{21}^{p}-1\right) \text {. }
$$


The fine grid convergence index is given by

$$
C G I^{\text {fine }}=\frac{F e_{21}^{a}}{r_{21}^{p}-1}
$$

where the safety factor is $F=1.25$ according to recommendations from NPARC (NPARC Alliance CFD Verification and Validation Web Site, 2018) and $e_{21}^{a}$ is the approximate error for the fine grid solution given by

$$
e_{21}^{a}=\left|\frac{\phi_{1}-\phi_{2}}{\phi_{1}}\right|
$$

An evaluation of the GCI analysis for drag estimates returned by the CGI analysis for the front nose refinement is given in Table 9.

\begin{tabular}{|c|c|c|c|c|c|}
\hline Refinement & $r_{12}$ & $r_{23}$ & $p$ & $\phi_{\text {ext }}$ & $C G I^{\text {fine }}$ \\
\hline$C_{d}$ & 2.05 & 1.95 & 2 & 0.329 & $2 \%$ \\
\hline
\end{tabular}

Table 9: Estimates of CGI parameters for lift and drag returned from the finest nose refinement.

Based on a CGI evaluation of the refined nose region, the evaluation of the drag is second order in space. However, the estimated exact solution shows a 2.7 percent difference to the experiment. This lies within the EN 14067-6 standard and, coupled with the estimated CGI index, suggests that results based on this mesh are acceptable for certification purposes. While Table 9 suggests that further refinement in the nose region may be of minimal benefit, it is clear that a more detailed evaluation of the CGI analysis for this problem should probably require more than three meshes in view of the variations observed for the underflow and wake regions.

In Table 7 the integral drag for a mesh with both refined underflow and front nose regions is presented. Also shown is the drag predicted on a mesh with both derefined underflow and front nose regions. On the basis of the results shown in this table wake refinements are not included in a combined refinement analysis since there is little variation in drag between the three levels of wake refinement. The result returned from the combined derefined underflow and front nose mesh $\left(M_{-1}^{F N+U f}\right)$ differs little from the solutions returned by the individual derefined nose and underflow solutions. The computed drag estimate from the mesh with the refined underflow and nose regions $\left(M_{1}^{F N+U F}\right)$ under-predicts the experiment by 2.4 percent, still satisfying the EN 14067-6 requirement. This result presents a slight degradation of the estimation returned by the finest nose refinement while considerably improving the drag estimate returned from the finest underflow refinement. Surface mesh resolution for the train nose is a dominant variable in controlling the quality of drag estimations by CFD.

\subsection{Surface Pressure Distributions}

In this section the influence of the mesh refinement strategy on vehicle surface pressure distributions along the symmetry axis $(x, 0, z) \mathrm{m}$ is discussed. Surface pressure distributions that are returned by all refinements along the model's roof are identical, as seen in Figure 10a. The combination of a relatively uniform wall-normal resolution and a constant on-flow velocity act, together with a well resolved reference mesh, to make the roof surface pressure distribution along the centerline essentially independent of the mesh refinement strategy. Note that the oscillation in the pressure at $\mathrm{x}=-0.7 \mathrm{~m}$ corresponds to a small bump in the surface geometry at this position. The underside surface pressure distributions are not significantly influenced by the refinements until the underflow starts to interact with the down flow over the train's rear head. In Figure 10b the influence of the first mounting post on the centerline distribution is illustrated. After the disturbance from the wake shed by the post, the surface pressure becomes independent of the mesh refinement. Differences in the wake region are due to reduced control volume sizes for the underfloor refinement and to changes in the underflow arising from the front nose refinement. Changes in the character of the underflow due to the nose refinement are seen in Figure 10c, where the magnitude of the pressure coefficient variations due to the wakes attached to the mounting posts are larger than those returned from the underflow and wake refinements. As the underflow enters the wake region the surface pressure contours (Figure 10d) demonstrate some slight variations. Pressure recovery is slightly improved at the rear floor region due to effect of the underflow refinement. This may be due to the influence of a reduction in numerical dissipation for the refined underflow mesh region on the computed pressure gradients. 


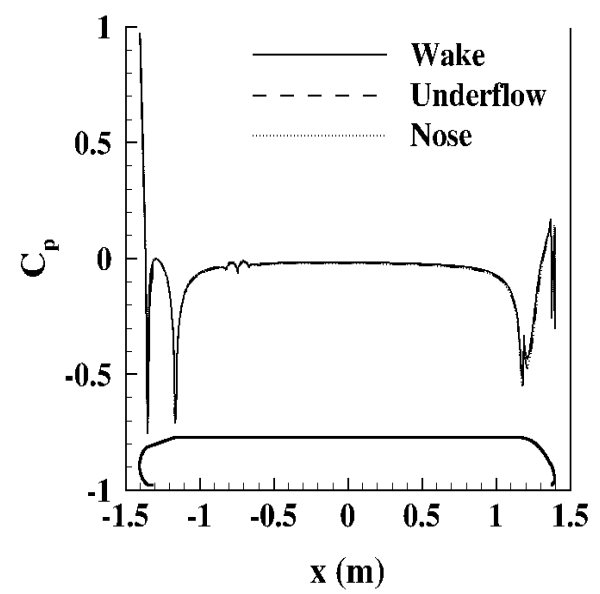

(a) Roof

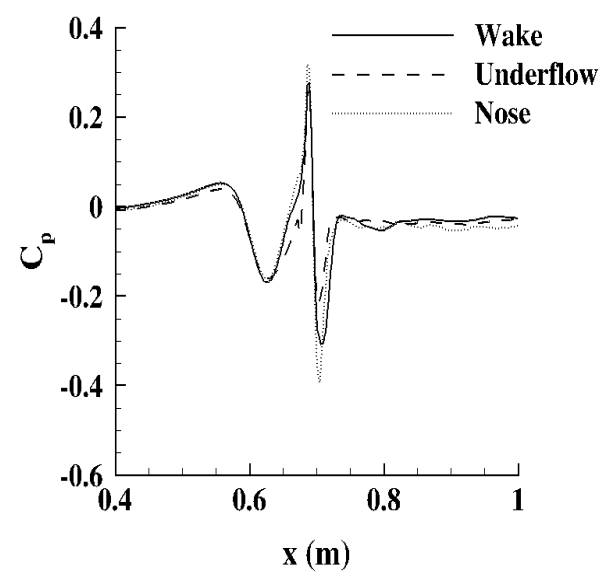

(c) Second mounting Pylon

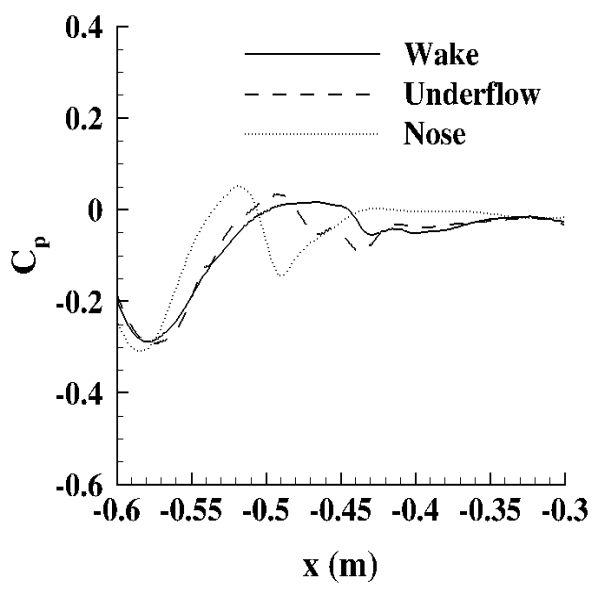

(b) First mounting pylon

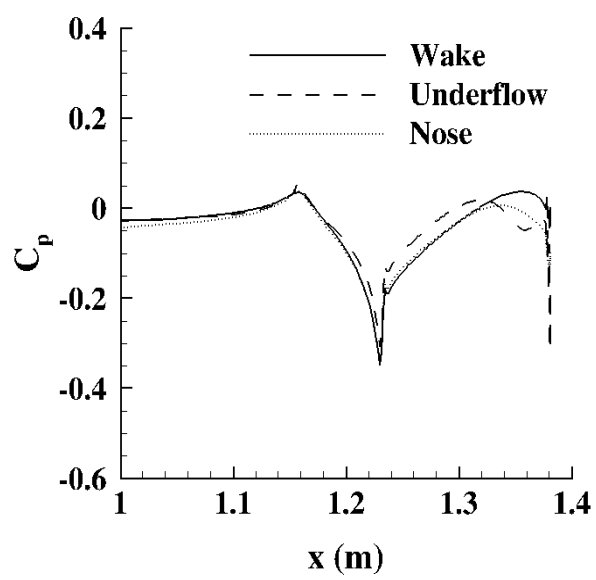

(d) Rear head

Figure 10: Surface pressure contour plots along the symmetry axis at $(x, 0, z) \mathrm{m}$ on the finest mesh refinements. Note: The contour of the train is provided in (a) to assist in interpretation of the surface pressure data.

\subsection{PIV Measurements in the Wake}

Figures 11 and 12 present PIV data in the orthogonal plane surfaces $S 1(x, y)$ and $S 2(x, z)$ respectively. The spatial resolution of the PIV system is $3.5 \mathrm{~mm}\left(0.03 L_{b}\right)$. The estimation error for the mean velocity at a point is bounded approximately by the measured RMS at the point and by the resolution of the PIV camera system. The measured RMS, normalized on the freestream velocity, varied from 20 percent for near-wall and wake regions to less than 5 percent elsewhere. The magnitude of the in-plane velocity fields are given by the following two equations for $S 1$ and $S 2$ :

$$
\begin{aligned}
& U_{x y}=\frac{\sqrt{u^{2}+v^{2}}}{U_{\infty}}, \\
& U_{x z}=\frac{\sqrt{u^{2}+w^{2}}}{U_{\infty}} .
\end{aligned}
$$

Here $u, v$, and $w$ are the (time-averaged) velocity components. Figure 11 compares both the PIV and computed in-plane velocity magnitudes and directions in the $x-y$ plane for the finest mesh refinements. The plots exhibit qualitative differences against the PIV data. There is little apparent variation between the CFD solutions which underestimate the extent of the trailing vortices and the effect these vortices have on the mean field through unsteady mixing. A direct consequence is that the velocity magnitude in the wake region is over-predicted. The main features in the PIV data are reproduced in the CFD. 


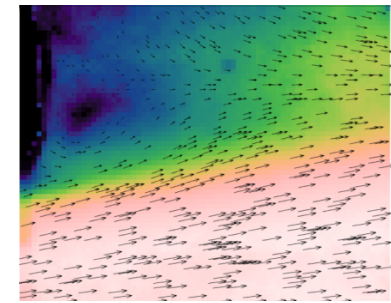

(a) PIV

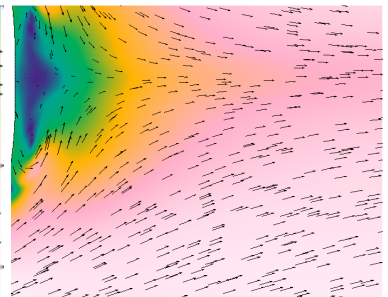

(b) Mesh $M_{1}^{W}$

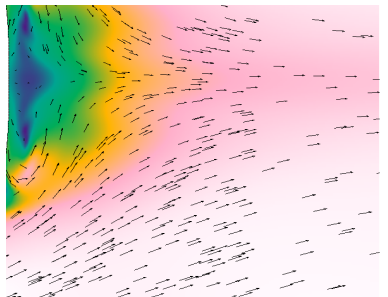

(c) Mesh $M_{1}^{U F}$

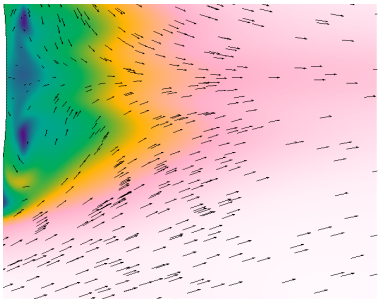

(d) Mesh $M_{1}^{F N}$

$\begin{array}{llllllllllllll}0 & 0.1 & 0.2 & 0.3 & 0.4 & 0.5 & 0.6 & 0.7 & 0.8 & 0.9 & 1\end{array}$

(e) Contour levels

Figure 11: Comparison for finest refinements of $U_{x y}$ in the $S_{1}$ plane.

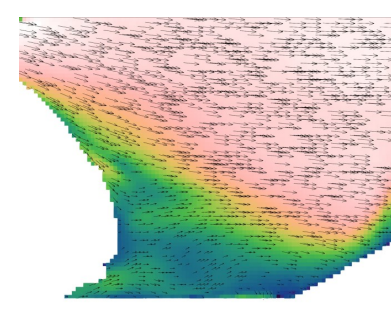

(a) PIV

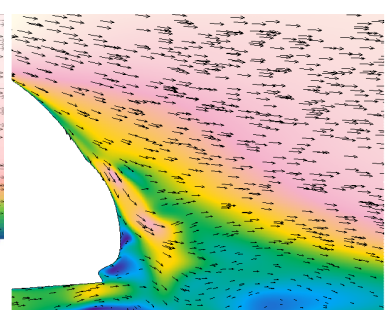

(b) Mesh $M_{1}^{W}$

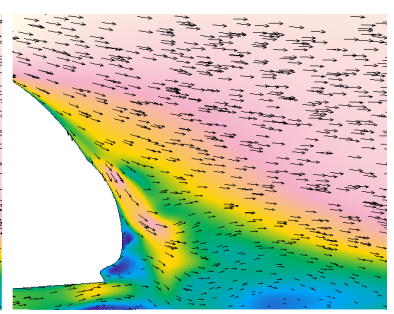

(c) Mesh $M_{1}^{U F}$

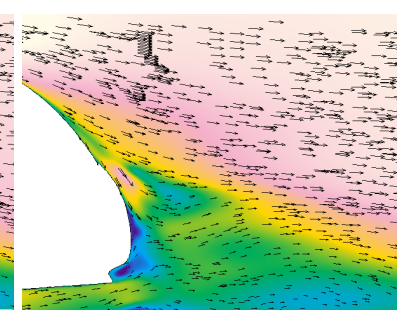

(d) Mesh $M_{1}^{F N}$

$\begin{array}{llllllllllll}0 & 0.1 & 0.2 & 0.3 & 0.4 & 0.5 & 0.6 & 0.7 & 0.8 & 0.9 & 1\end{array}$

(e) Contour levels

Figure 12: Comparison for finest refinments of $U_{x z}$ in the $S_{2}$ plane.

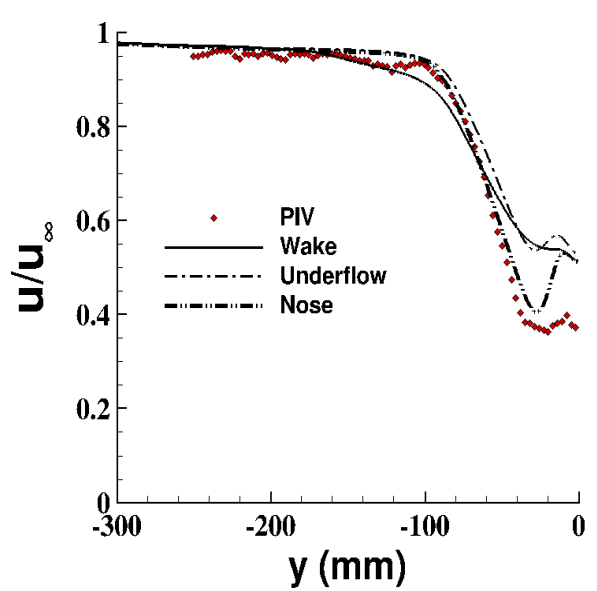

(a) Profile of $u$ at $X=(1.5, y, 0.12) \mathrm{m}$ along the $S_{1}$ plane.

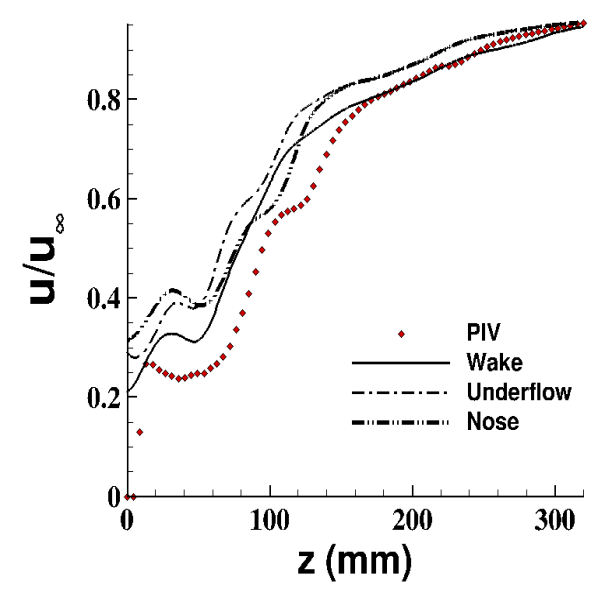

(b) Profile of $u$ at $X=(1.5,-0.05, z) \mathrm{m}$ along the $S_{2}$ plane.

Figure 13: Comparison of the $u$ velocity component returned from the refined meshes against PIV data.

Figure 12 compares both the PIV and computed in-plane velocity magnitudes and directions in the $\mathrm{x}$-z plane for the finest mesh refinements. All plots exhibit qualitative differences against the PIV. The solution returned on the finest nose refinement shows the best qualitative agreement against the PIV data. In all of these figures a "jet" of high momentum fluid starts at approximately halfway up the train nose. This structure is generated by the trailing vortex system. For the underflow and wake refinements this region is significantly over-predicted and penetrates 
into the existing underflow. Figure 13 illustrates contours of the $u$-velocity component along cutting lines in the $S_{1}$ and $S_{2}$ planes. The CFD and PIV data are in good agreement far from the wake region but notable differences are seen in the wake region. The CFD overestimates the measured $u$ component but the trends seen in the PIV data are largely replicated. The flattening of the velocity profile about the wake centerline is essentially predicted by all CFD calculations, but unsteady flow resolving methods are needed to better match the experiment results in the wake. Despite the differences apparent in comparisons between experiment and CFD, the previous section demonstrates that the wake flow plays a lower order role in the vehicle drag and does not need to be rigorously addressed.

\section{Computational Efficiency}

Calculations were performed on the DLR's SCART2 HPC cluster located in Göttingen, Germany. The SCART2 cluster used during this work is a distributed memory parallel computer with 256 nodes. Each node is equipped with two Intel Ivy-Bridge (10-Core) processors and a 64Gb high speed memory (1866 MHz) card. The SuSE Linux Enterprise server (SLED11 SP3) is used as the operating system on each node. The system supports various flavors of OpenMPI built against the GNU 4.3 and Intel compilers. This work used a version of OpenFOAM built using the system OpenMPI-1.4.3 libraries and GCC compiler. Further details of the SCART cluster are proprietary but can be found in Lapeira et al. (L. Lapeira and B. Wiegers and T.Alrutz, 2015) by request. Meshes were of the order of 40-80 million points and a domain decomposition method using 80-180 domains was applied to the solution of the discrete linear system. A converged solution of the equations is assumed when the convergence criterion specified in Appendix $\mathrm{C}$ were satisfied. The number of points per domain was dependent upon available resources (the SCART2 cluster is heavily utilized) and data exchange requirements (the parallel speedup in OpenFOAM is approximately linear for the number of processors used but the available number of processes is dependent on the cluster usage and it was not possible to operate with a fixed number of processors). The solver required approximately 3.3e-6 seconds per iteration per mesh point per domain for a 42.2 million point grid decomposed into 120 domains. A satisfactory load balancing across partitions was achieved with the OpenFOAM partitioning library.

Initial flow fields were generated initially by a potential flow method - this step assists in reducing initial transient effects which generally require a significantly larger computational time to dampen out. Note that OpenFOAM provides functionality to map solutions across meshes but we did not use this method as a means to further reduce the initialization time required. There may be an advantage in using mapping technique to reduce overall computational time but this question is not addressed in this work. The use of domain decomposition methods significantly reduced the memory requirement per domain, as well as reducing the computational time required per iteration. However, as noted previously in this section, the number of available computational nodes was dependent on cluster usage. Using an estimate of the convective time scale (based on the reference velocity and model width), an unsteady convective time unit can be computed in 6 hours wall time on the assumption that a time step of one hundredth of the convective time step is chosen. Based on experience with other geometries, at least several hundred convective time units are required in order to properly assess second-order flow correlations. This requires significant computational effort. For the steady calculations presented in this paper results are obtained in considerable shorter time. The results in this paper partially justify the fact that industrial practitioners favor steady methods albeit at some cost in modeling accuracy.

\section{Conclusions}

A numerical grid refinement study, based on a RANS modeling approach, has been conducted and compared against experimental data on the flow about a simplified short-length model train. The front nose, underflow and wake regions about the train were independently refined. The resolution of the nose region plays a major role for an accurate estimation of the drag force. The effect of mesh refinement in the wake and underflow regions did not return any clear benefits. While this observation partially demonstrates a limitation of RANS methods in separated flows, the principal concern for RANS based industrial methods should be in properly modeling the pressure drag that arises from the front of the vehicle. The interaction of the underflow with down-flow over the rear train head and the wake is highly complex and presents a challenge to modern industrial turbulence modeling. The results obtained in this work suggest accurate modeling of this region appears to be of secondary importance for useful drag estimations. Preliminary guidelines for adequate mesh resolution with regard to the question of drag assessment have been given in the paper but it should be again noted that these recommendations are formally valid for RANS solutions of vehicles of equivalent geometries and Reynolds number. 


\section{A Justification for an incompressible analysis}

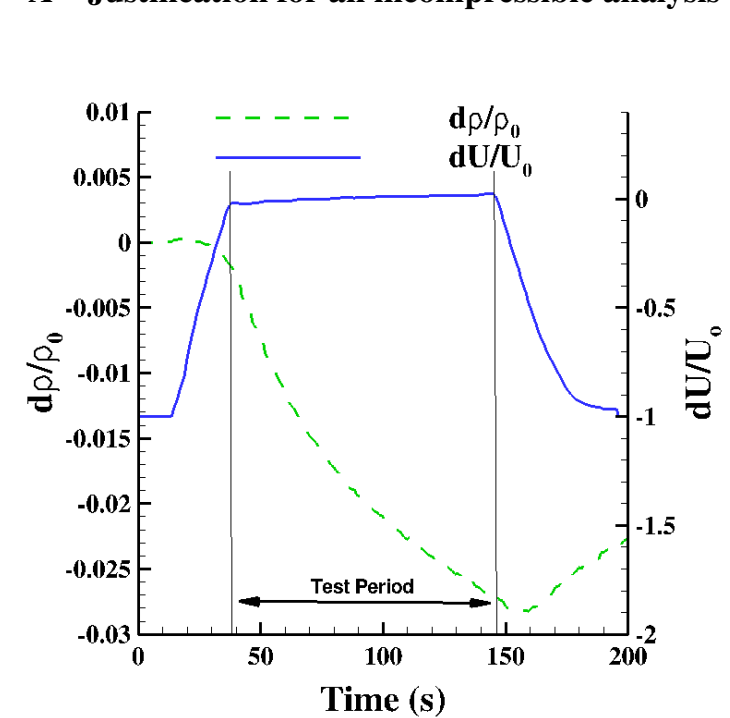

Figure A.1: Profiles of density and velocity changes over a typical test period of 100 seconds. Changes in density and velocity are scaled with respect to reference values of density $\left(\rho_{o}\right)$ and the mean bulk velocity $\left(U_{o}\right)$.

Bombardier Transport provided both significant contributions to the development of the wind tunnel model and significant technical input. M.M. Fragner was funded through an agreement between the DLR and Bombardier Transport GmbH.

\section{Appendices}

Figure A.1 illustrates the variation in density and stream-wise velocity over a single force measurement run at the operating conditions given in Table 1. Combination of the equation for momentum conservation for a steady inviscid flow (assuming no external forces)

$$
\rho U d U=-d P
$$

with the isentropic flow relation

$$
d P=\gamma \frac{P}{\rho} d \rho
$$

where $\gamma$ is the ratio of specific heats at constant pressure and constant volume, yields the following relationship relating Mach number to the change in density

$$
-M^{2} \frac{d U}{U}=\frac{d \rho}{\rho}
$$

From inspection of CFD and PIV data the ratio $d U / U$ is set to an upper limit of $\sim 0.75$. The density change over a typical run is estimated to be of $\sim 0.025$ from Figure A.1 so that the Mach number limit for this density change is $\sim 0.2$. From Table 1 the Mach number in the wind tunnel experiment is $\sim 0.2$ so that an assumption of incompressibility at tunnel conditions is justified, albeit close to the upper limits of incompressibility.

\section{B The Menter-SST Turbulence Model}

The Menter-SST (Shear-Stress Transport) model (Menter, 1994) forms the basis of the implementation in OpenFOAM. This model, using a linear eddy viscosity model based on the Boussinesq assumption, is often used for industrial applications.

$$
\tau_{i j}=2 \mu_{t}\left(S_{i j}-\frac{1}{3} \frac{\partial u_{k}}{\partial x_{k}} \sigma_{i j}\right)-\frac{2}{3} \rho \sigma_{i j}
$$


Note that for constant density flows $\frac{\partial u_{k}}{\partial x_{k}}=0$. It should be noted that solution algorithm used for the pressure equation fixes a divergence free velocity field for the converged velocity field. The symmetric second-order velocity gradient tensor $S_{i j}$ is given by

$$
S_{i j}=\frac{1}{2}\left(\frac{\partial u_{j}}{\partial x_{i}}+\frac{\partial u_{i}}{\partial x_{j}}\right) .
$$

The conservative form of this two-equation turbulence model is written below as

$$
\begin{aligned}
\frac{\partial(\rho k)}{\partial t}+\frac{\partial\left(\rho u_{j} k\right)}{\partial x_{j}}= & P-\beta^{\star} \rho k \omega+\frac{\partial}{\partial x_{j}}\left[\left(\mu+\sigma_{k} \mu_{t}\right) \frac{\partial k}{\partial x_{j}}\right] \\
\frac{\partial(\rho \omega)}{\partial t}+\frac{\partial\left(\rho u_{j} \omega\right)}{\partial x_{j}},= & \frac{\gamma}{\nu_{t}} P-\beta \rho \omega^{2}+\frac{\partial}{\partial x_{j}}\left[\left(\mu+\sigma_{\omega} \mu_{t}\right) \frac{\partial \omega}{\partial x_{j}}\right], \\
& +2\left(1-F_{1}\right) \frac{\rho \sigma_{\omega 2}}{\omega} \frac{\partial k}{\partial x_{j}} \frac{\partial \omega}{\partial x_{j}} .
\end{aligned}
$$

Note that in this work the time derivative terms above dissappear since steady-state calculations are made. Production of turbulent kinetic energy $P$ is given by

$$
P=\tau_{i j} \frac{\partial u_{j}}{\partial x_{j}} .
$$

A production limiter (Menter, 1994) is implemented and $P$ in the $k$ equation is replaced by $\min \left(P, 20 \beta^{\star} \rho \omega k\right)$. The OpenFOAM version uses a value of 10 instead of 20 . The turbulent viscosity is modeled by

$$
\mu_{t}=\frac{\rho a_{1} k}{\max \left(a_{1} \omega, \Omega F_{2}\right)} .
$$

Note that $\Omega=\sqrt{2 W_{i j} W_{i j}}$ is the vorticity magnitude where

$$
W_{i j}=\frac{1}{2}\left(\frac{\partial u_{i}}{\partial x_{j}}-\frac{\partial u_{j}}{\partial x_{i}}\right) .
$$

The use of the vorticity magnitude in the above equation is based on dimensional reasoning, and a later form of the SST model (Menter et al., 2003) uses the strain invariant $S=\sqrt{2 S_{i j} S_{i j}}$ in place of the vorticity magnitude. All constants are blended between inner (1) and outer (2) constants, whereby inner constants are representative of boundary layer properties and the outer constants are representative of the farfield flow behavior. Simple linear blending is used via the following linear scheme

$$
\phi=F_{1} \phi_{1}+\left(1-F_{1}\right) \phi_{2} .
$$

Model closure is obtained by the application of the following equations:

$$
\begin{aligned}
F_{1} & =\tanh \left(\arg _{1}\right), \\
\arg _{1} & =\min \left[\max \left(\frac{\sqrt{k}}{\beta^{\star} \omega d}, \frac{500 \nu}{d^{2} \omega}\right), \frac{4 \rho \sigma_{\omega 2} k}{C D_{k \omega} d^{2}}\right], \\
C D_{k \omega} & =\max \left(2 \rho \sigma_{\omega 2} \frac{1}{\omega} \frac{\partial k}{\partial x_{j}} \frac{\partial \omega}{\partial x_{j}}, 10^{-20}\right), \\
F_{2} & =\tanh \left(\arg _{2}^{2}\right), \\
\arg _{2} & =\max \left(2 \frac{\sqrt{k}}{\beta^{\star} \omega d}, \frac{500 \nu}{d^{2} \omega}\right),
\end{aligned}
$$

where $d$ is the distance from a field point to the nearest wall. Model constants are listed in Table B.1. Additional limiters within the OpenFOAM code were added to prevent accumulation of floating point errors and to prevent division by zero. Terms like $\omega d$ were computed as $\max \left(\omega d, \omega_{\min } d_{\text {min }}\right)$. Note that $\omega_{\text {min }}$ and $d_{\text {min }}$ are set to the smallest representable floating point number $\epsilon \approx O\left(10^{-16}\right)$ for double precision calculations. 


$$
\begin{array}{ccc}
\gamma_{1}=\frac{\beta_{1}}{\beta^{\star}}-\frac{\sigma_{\omega 1} \kappa^{2}}{\sqrt{\beta^{\star}}} \quad \gamma_{2}=\frac{\beta_{2}}{\beta^{\star}}-\frac{\sigma_{\omega 2} \kappa^{2}}{\sqrt{\beta^{\star}}} \\
\sigma_{k 1}=0.85 & \sigma_{\omega 1}=0.5 & \beta_{1}=0.075 \\
\sigma_{k 2}=1.0 & \sigma_{\omega 2}=0.856 & \beta_{2}=0.0828 \\
\beta^{\star}=0.09 & \kappa=0.41 & a_{1}=0.31 \\
\hline
\end{array}
$$

Table B.1: SST model coefficients

\section{Numerical Simulation Tools}

The numerical equations describing the evolution of the fluid have been solved using the open source OpenFOAM libraries (Weller et al., 1998). We use a commercial version of this library (HELYX-Core 2.2_engysEdition-1.1) provided by ENGYS Ltd (for further details on ENGYS the reader is referred to the web site www.engys.com) for both the numerical solver and for mesh generation (snappyHexMesh). The steady state version of the solver is used. Parameter settings for the solver are related mostly to the choice of the discretization operator. Bounded gradient limiters are chosen for turbulence variables in order to prevent overshoots in the gradient estimation and to assist in maintaining stability, as can be surmised by inspection of the following fragment from the fivschemes dictionary.

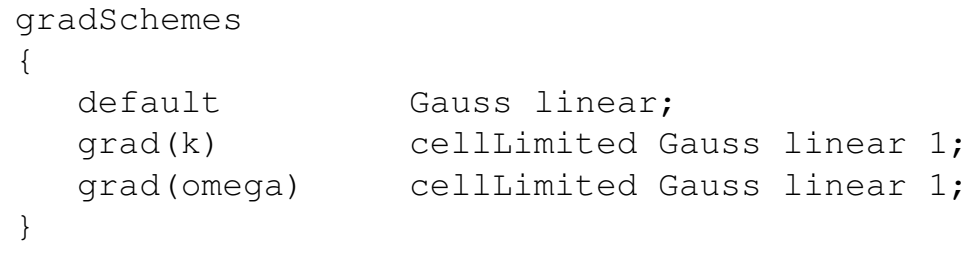

A second-order upwind-biased unbounded operator has been chosen for inviscid flux terms, while a second-order central differencing operator is used on viscous fluxes.

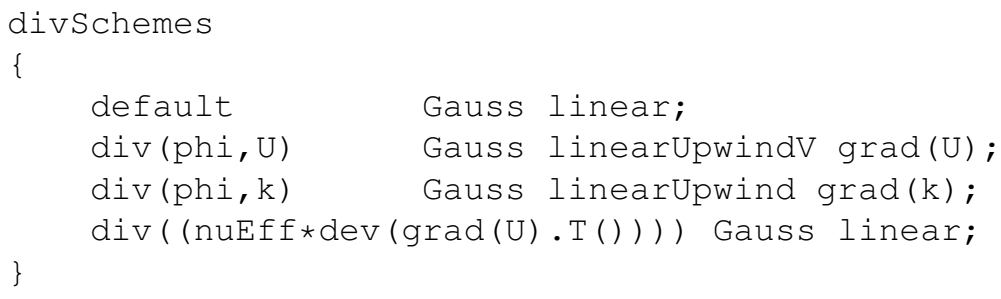

Surface normal gradients are estimated with a scheme that is second order for orthogonal meshes. If orthogonality of the mesh decreases, explicit corrections are applied which can drive the solution into an unstable regime. Potential instabilities are stabilized by applying a limiter as follows.

$$
\text { default limited } 0.333 \text {; }
$$

The Laplacian operator is a combination of the divergence and gradient operators and is descretized via Gauss's theorem. Again a limiter is used to suppress instabilities as follows:

default Gauss linear limited 0.333;

The Semi-Implicit Method for Pressure Linked Equations (Patankar and Spalding, 1972) is used to solve the discrete equations. The equations were solved sequentially until convergence, which was assumed when residuals reduced to a level below a threshold of $O\left(10^{-6}\right)$ with the divergence of the velocity field being less than $10^{-10}$. The linear system of equations arising from the implicit temporal discretization contains a high degree of sparsity and was solved iteratively using a preconditioned conjugate gradient method. Diagonal based incomplete Cholesky pre-conditioning was applied to the pressure correction equation while a preconditioned bi-conjugate method with a simplified diagonal based incomplete LU pre-conditioner is used for the other dependent variables.

\section{Mesh Generation Dictionary}

Useful parameter settings of the snappyHexMesher control dictionary are given in the following dictionary fragment. 


\section{- Initialization}

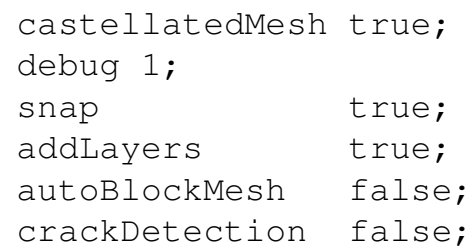

- castellatedMeshControls (Refined Surfaces)

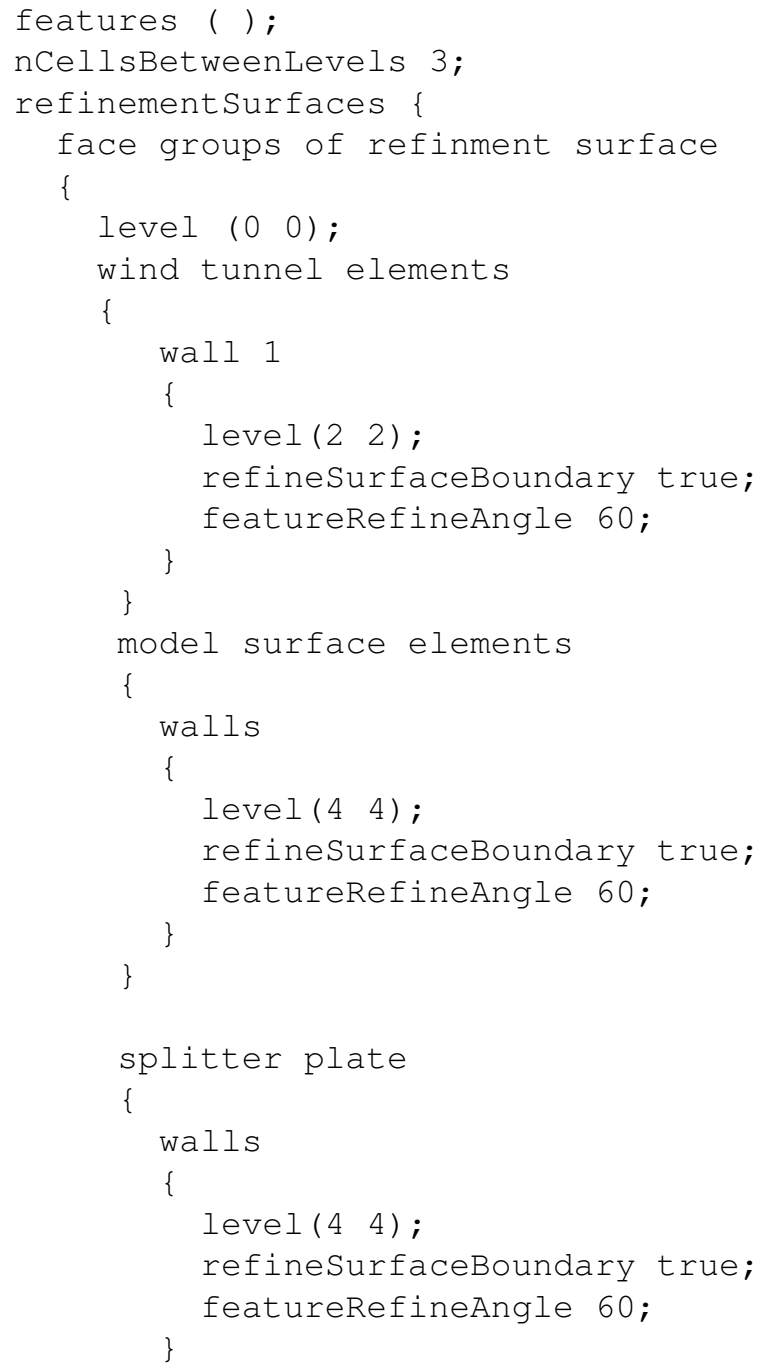

- castellatedMeshControls (Refinement Regions)

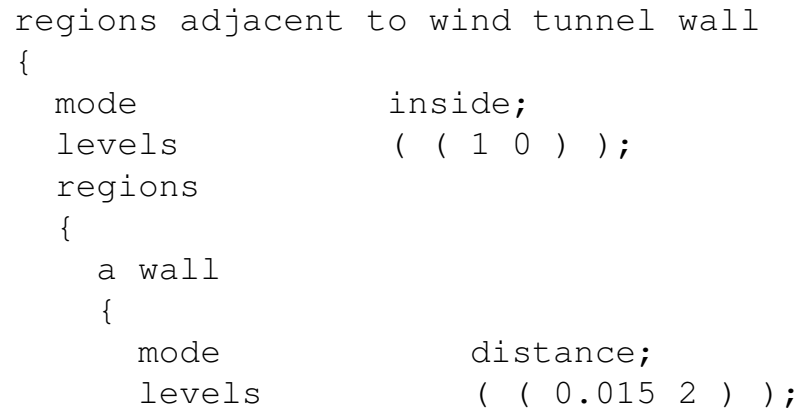




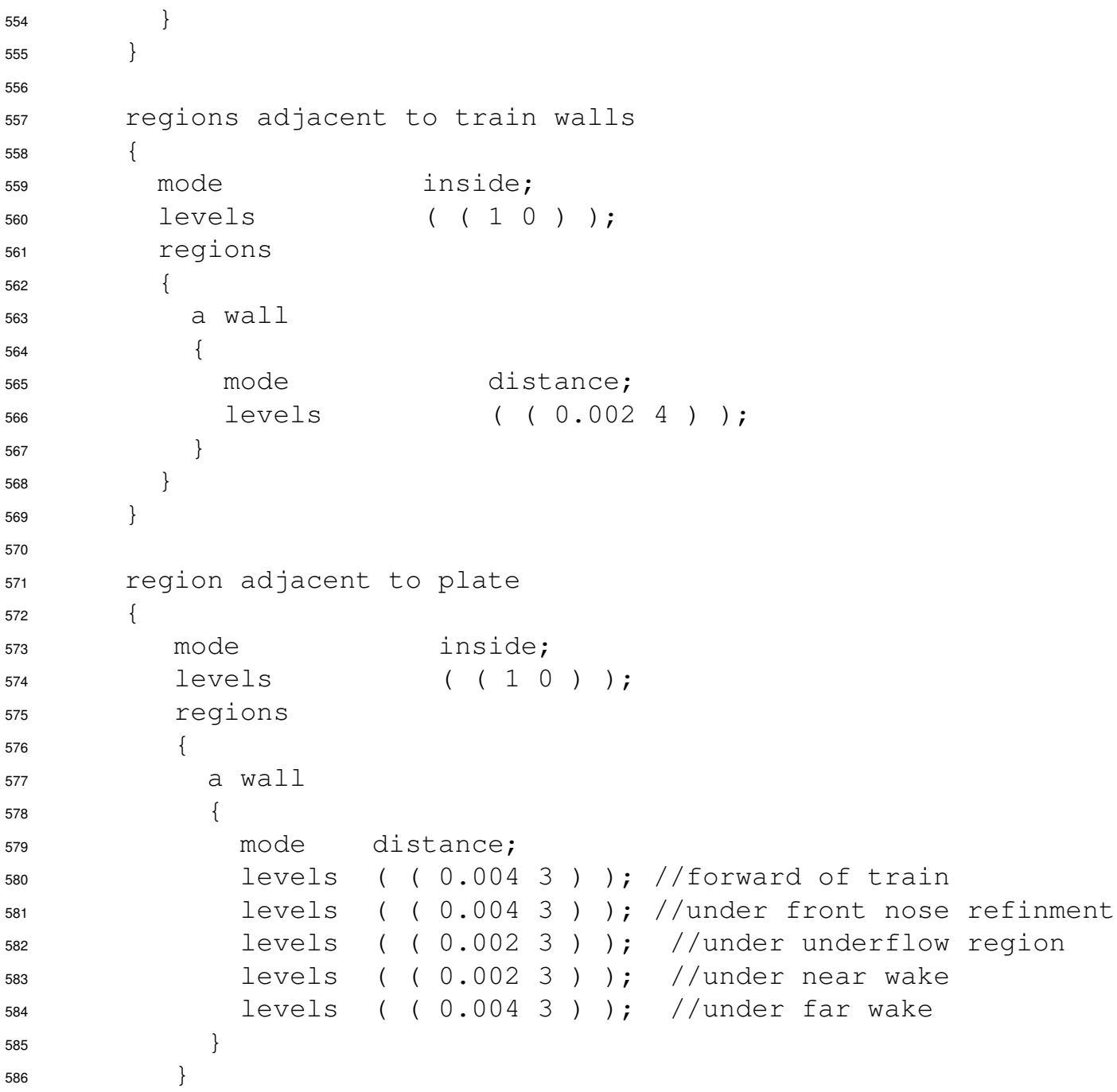

- Castellation Controls

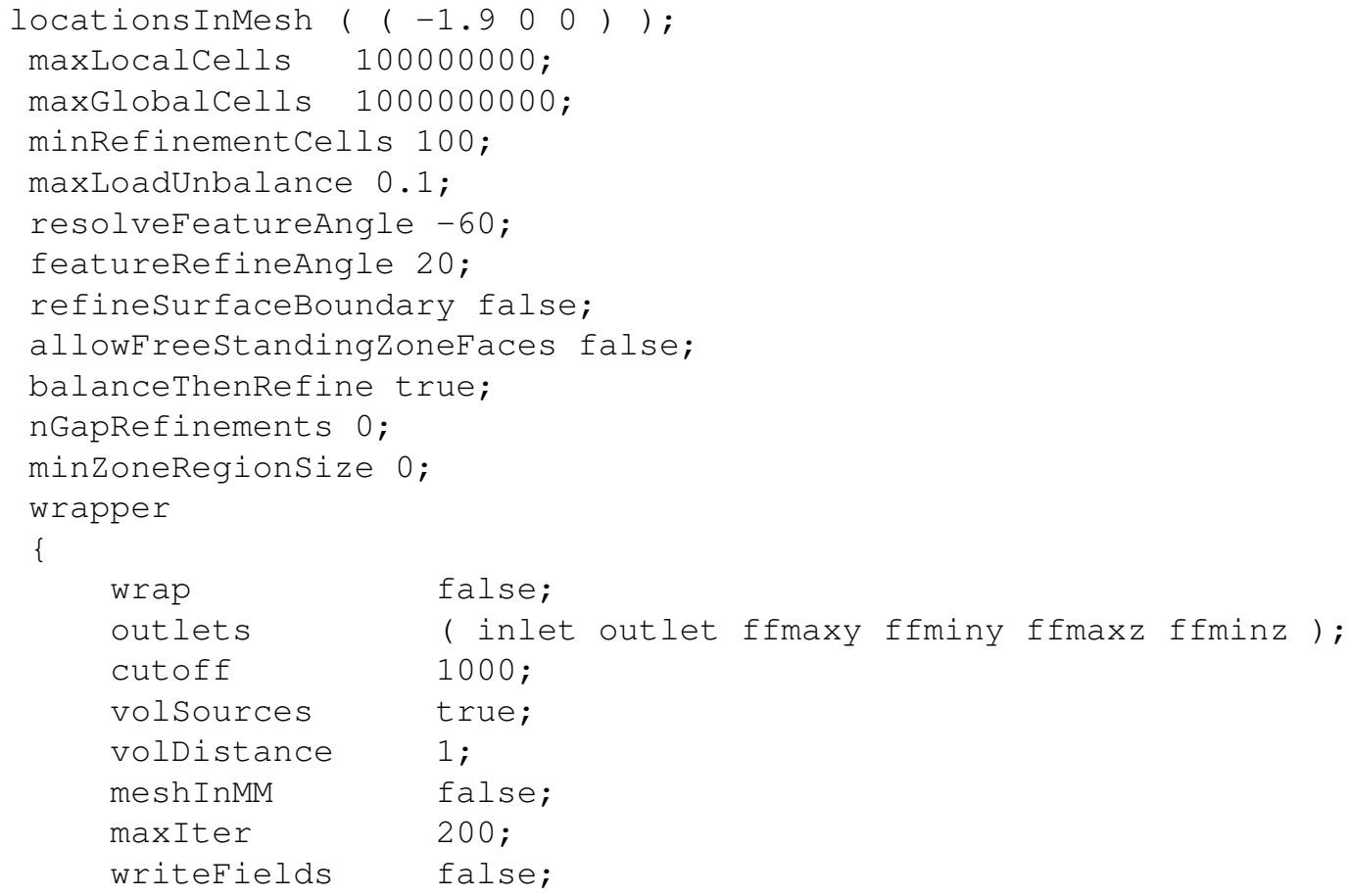


- Layer Controls

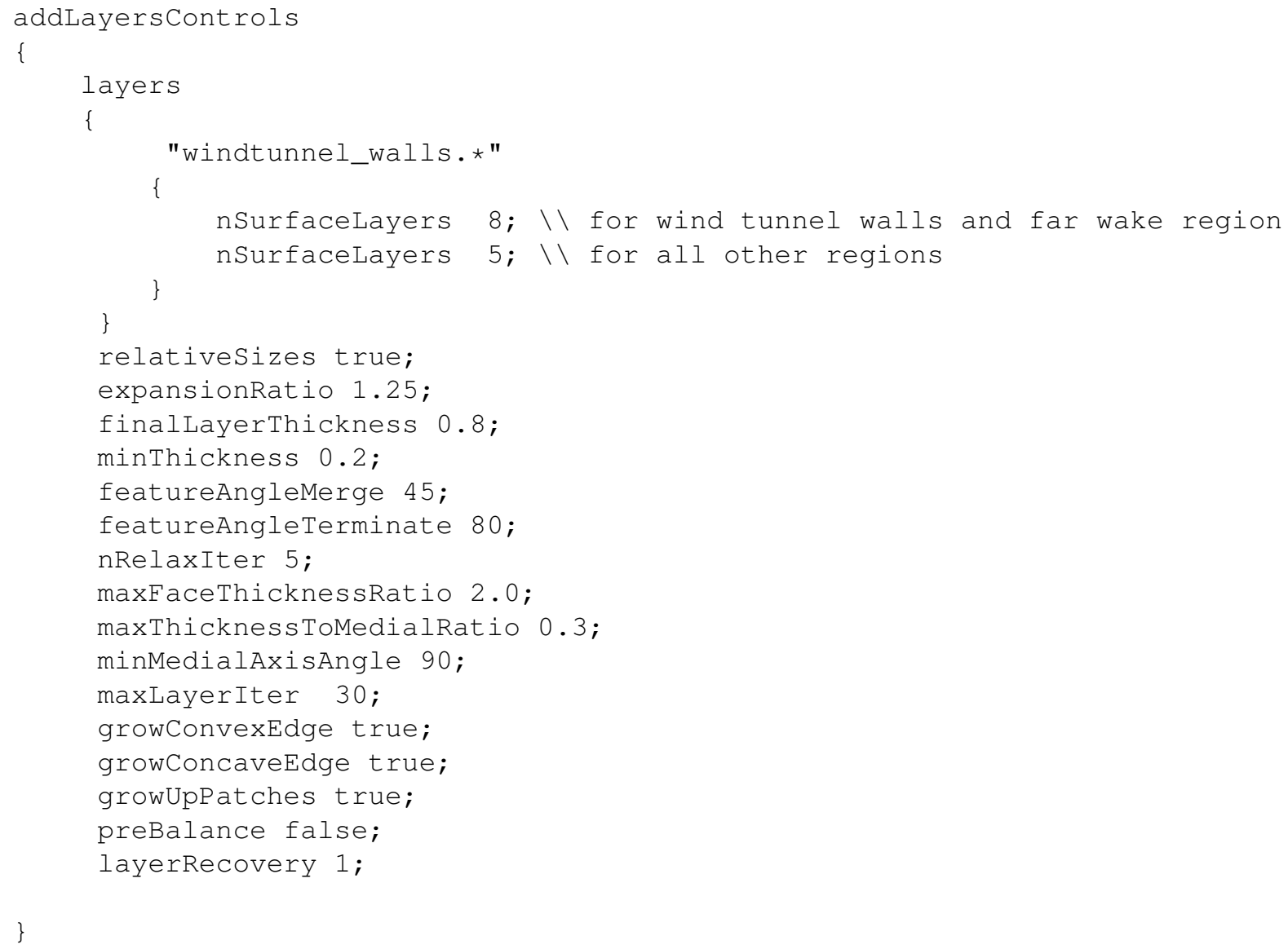

- Mesh quality controls

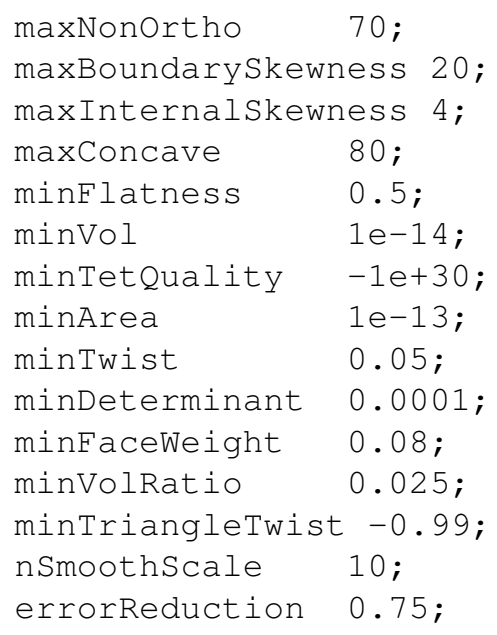

A typical mix of control volume type numbers obtained for $M_{3}^{F N}$ is as follows; hexahedra (115031790), prisms (488105), wedges (41533), pyramids (155), tet wedges (4712), tetrahedra (45003) and polyhedra (1584361). It would be ideal for the characterization of numerical dissipation effects to use only one element type for volume and and one type for surface discretizations but we were unable to achieve this for a hybrid mesh with the OpenFOAM mesher. This may be possible with more recent versions but this has not been explored. A pure tetrahedral mesh satisfies this requirement but the attached boundary layers requires high resolution in the wall-normal direction 
only. A significantly larger number of tetrahedral volumes of aspect ratio $\mathrm{O}(1)$ would be required to resolve wallnormal regions in comparison to hexahedral or prismatic volumes where aspect ratios in directions tangent to the wall can be of $\mathrm{O}(1000)$ or larger.

Anderson, D. A. and Tannehill, J. C., editors (1984). Computational Fluid Mechanics and Heat Transfer. McGraw Hill Book Company, New York.

Baker, C. (2010). The flow around high speed trains. Journal of Wind Engineering and Industrial Aerodynamics, 98. $277-298$

Baker, C. (2014). A review of train aerodynamcics: Part 1 - Fundamentals. The Aeronautical Journal, 118. no. 1201.

DB Netz AG (2010). Richtlinie 807.04 Bautechnik, Leit-, Signal- und Telekommunikationstechnik. Ausgewählte Maßnahmen und Anforderungen and das Gesamtsystem Fahrweg/Fahrzeug Aerodynamik/Seitenwind. Technical report, DB Netz AG, Frankfurt.

Deiterding, R. (2011). Block-structured adaptive mesh refinment - theory, implementation and application. In ESAIM: Proceedings, pages 34-97.

Fragner, M. M. (2015). Presentation: Steering Board Meeting Best Drag Project. Validatation results presented to steering committee of Bombardier.

Fragner, M. M. and Deiterding, R. (2016). Investigating cross-wind stability of high speed trains with large-scale parallel CFD. Int. J. Comput. Fluid Dynamics, 30(6):402-407.

Fragner, M. M., Weinman, K. A., Deiterding, R., Fey, U., and Wagner, C. (2015). Comparison of industrial and scientific CFD approaches for predicting cross wind stability of the NGT2 model train geometry. Int. J. Railways Techn., 4(1):1-18.

H. N. Hemida and S. Krajnovic (2010). LES Study of the influence of the nose shape and yaw angles on flow structures around trains. Journal of Wind Engineering and Industrial Aerodynamics, 98(1):34-46.

Haff, J., Richard, U., Kowalski, T., Loose, S., and Wagner, C. (2012). Wind Tunnel Experiments with a HighSpeed Train model subject to Cross-Wind Conditions. In J.Pombo, editor, Proceedings on the first international conference on Railway Technology:Research, Development and Maintenance, volume 24. Civil-Comp Press, Stirlingshire,Scotland.

HELYX (2014). HELYX@ Core User Reference Guide. Technical report, ENGYS Ltd.

I. B. Celik and U. Ghia and P. J. Roache and C. J. Freitas and H. Coleman and P. E. Raad (2008). Proceedure for Estimation and Reporting of Uncertainly Due to Discretization in CFD Applications. Journal of Fluids Engineering, 130.

J. Morden and H.Hemida and C. Baker (2015). Comparison of RANS and detached eddy simulation results to wind-tunnel data for the surface pressures upon a class 43 high-speed train . Journal of Fluids Engineering, 137(4). DOI:10.1115/1.4029261.

L. Lapeira and B. Wiegers and T.Alrutz (2015). SCART 2-Short UsersGuide Version 1.0. Technical report, TSystems for Research GmbH, Köln, Germany.

L.F. Richardson (1910). The approximate arithmetical solution by finite differences of physical problems involving Differential Equations, with an application of the stresses in a masonary dam. Philos. Trans. R. Soc. London, 210:307-357.

Menter, F. R. (1994). Two-Equation Eddy-Viscosity Turbulence Models for Engineering Applications. AIAA Journal, Vol. 32(8),:1598-1605.

Menter, F. R., Kuntz, M., and Langtry, R. (2003). Ten Years of Industrial Experience with the SST Turbulence Model. Turbulence, Heat and Mass Transfer 4, pages 625-632.

Muld, T. W. (2012). Slipstream and flow structures in the near wake of high-speed trains. Technical report, Royal Insititute of Technology, Department of Aeronautical and Vehicle Engineering, Stockholm, Sweden.

NPARC Alliance CFD Verification and Validation Web Site (2018). Examining Spatial (Grid) Convergence. https://www.grc.nasa.gov/www/wind/valid/tutorial/spatconv.html.

Orellano, A. (2010). Aerodynamics of High Speed Trains. Vehicle Aerodynamics lecture, Stockholm KTH.

P. J. Roache (1998). Guide for the verification and validation of Computational Fluid Dynamics Simulations. AIAA G-077-1988.

Patankar, S. V. and Spalding, D. B. (1972). A calculation procedure for heat, mass and momentum transfer in three-dimensional parabolic flows. Int. J. of Heat and Mass Transfer, 15(10):1787-1806.

Sima, M., Gurr, A., and Orellano, A. (2008). Validation of CFD for the flow under a train with 1:7 scale wind tunnel measurements. In ERCOFTAC, editor, BBAA IV International Colloquium on Bluff Bodies Aerodynamics and Applications, volume 9, pages 1638-1649. 
Spalart, P. (2001). Young-Person's Guide to Detached-Eddy Simulation Grids. Technical report, NASA/CR-2001 211032.

W. Haase and M.Braza and A. Revell (2009). DESider A European Effort on Hybrid RANS-LES Modelling: Results on the European-Union Funded Project, 2004-2007. Springer Publishing Company, 1st edition. ISBN:3540927727 9783540927723.

Weinman, K. A., Fey, U., Loose, S., Wagner, C., Deiterding, R., and Fragner, M. M. (2013). Comparison between CFD and Wind Tunnel experiment for slender bodies of aspect-ratio $\mathrm{O}(1)$ in the presence of cross-wind. In 10th World Conference on Railway Research.

${ }_{228}$ Weller, H. G., Tabor, H., Jasak, H., and Fureby, C. (1998). A tensorial approach to computational continuum mechanics using object-oriented techniques. Computers in Physics, 12.

Wilcox, D. C. (2006). Turbulence Modeling for CFD. DCW Industries, La Canada, California, USA, 3rd edition edition. ISBN 978-3-540-92772-3. 